\title{
Stellar Orbits Around the Galactic Center Black Hole
}

\author{
A. M. Ghez ${ }^{1}$, S. Salim, S. D. Hornstein, A. Tanner, J. R. Lu, M. Morris, E. E. Becklin, G. \\ Duchêne \\ UCLA Division of Astronomy and Astrophysics, Los Angeles, CA 90095-1574 \\ ghez, samir, seth, tanner, jlu, morris, becklin, duchene@astro.ucla.edu
}

\begin{abstract}
We present new diffraction-limited images of the Galactic Center, obtained with the W. M. Keck I 10-meter telescope. Within 0'.4 of the Galaxy's central dark mass, 17 proper motion stars, with $K$ magnitudes ranging from 14.0 to 16.8, are identified and 10 of these are new detections (6 were also independently discovered by Schödel et al. 2003). In this sample, three newly identified (S0-16, $\mathrm{S} 0-19$, and S0-20) and four previously known (S0-1, S0-2, S0-4, and S0-5) sources have measured proper motions that reveal orbital solutions.

Orbits are derived simultaneously so that they jointly constrain the central dark object's properties: its mass, its position, and, for the first time using orbits, its motion on the plane of the sky. This analysis pinpoints the Galaxy's central dark mass to within 1.3 mas (10 AU) and limits its proper motion to $1.5 \pm 0.5$ mas $\mathrm{y}^{-1}$ (or equivalently $60 \pm 20 \mathrm{~km} \mathrm{~s}^{-1}$ ) with respect to the central stellar cluster. This localization of the central dark mass is consistent with our derivation of the position of the radio source Sgr $A^{*}$ in the infrared reference frame ( \pm 10 mas), but with an uncertainty that is a factor 8 times smaller, which greatly facilitates searches for near-infrared counterparts to the central black hole. Consequently, one previous claim for such a counterpart can now be ascribed to a close stellar passage in 1996. Furthermore, we can place a conservative upper limit of 15.5 mag on any steady-state counter-part emission. The estimated central dark mass from orbital motions is $3.7( \pm 0.2) \times 10^{6}\left(\frac{R_{o}}{8 k p c}\right)^{3} M_{\odot}$; this is a more direct measure of mass than those obtained from velocity dispersion measurements, which are as much as a factor of two smaller. The Galactic Center's distance, which adds an additional $19 \%$ uncertainty in the estimated mass, is now the limiting source of uncertainty in the absolute mass. For stars in this sample, the closest approach is achieved by $\mathrm{S} 0-16$, which came within a mere $45 \mathrm{AU}\left(=0.0002 \mathrm{pc}=600 \mathrm{R}_{s}\right)$
\end{abstract}

\footnotetext{
${ }^{1}$ Institute of Geophysics and Planetary Physics, University of California, Los Angeles, CA 90095-1565
} 
at a velocity of $12,000 \mathrm{~km} \mathrm{~s}^{-1}$. This increases the inferred dark mass density by four orders of magnitude compared to earlier analyses based on velocity and acceleration vectors, making the Milky Way the strongest existing case for a supermassive black hole at the center of a normal type galaxy.

Well determined orbital parameters for these seven Sgr A* cluster stars provide new constraints on how these apparently massive, young ( $<10 \mathrm{Myr})$ stars formed in a region that seems to be hostile to star formation. Unlike the more distant He-I emission line stars - another population of young stars in the Galactic Center - that appear to have co-planar orbits, the Sgr A* cluster stars have orbital properties (eccentricities, angular momentum vectors, and apoapse directions) that are consistent with an isotropic distribution. Therefore many of the mechanisms proposed for the formation of the He-I stars, such as formation from a pre-existing disk, are unlikely solutions for the Sgr A* cluster stars. Unfortunately, alternative theories for producing young stars, or old stars that look young, in close proximity to a central supermassive black hole, are all also somewhat problematic. Understanding the apparent youth of stars in the Sgr A* cluster, as well as the more distant He I emission line stars, has now become one of the major outstanding issues in the study of the Galactic Center.

Subject headings: black hole physics - Galaxy:center — Galaxy:kinematics and dynamics — infrared:stars - techniques:high angular resolution

\section{Introduction}

The proximity of our Galaxy's center (8 kpc, Reid 1993) presents an opportunity to build a case for a supermassive black hole and to study the black hole's environment and its effects thereon with much higher spatial resolution than can be brought to bear on any other galaxy. The first hint of a central concentration of dark matter in the Milky Way came from radial velocity measurements of ionized gas located in a three-armed structure known as the mini-spiral, which extends from the center out to $1-3$ pc (Lacy et al. 1980). Concerns that the measured gas motions were not tracing the gravitational potential were quickly allayed by radial velocity measurements of stars, which are not susceptible to nongravitational forces (McGinn et al. 1989; Sellgren et al. 1990; Haller et al. 1996; Genzel et al. 1997). These early, low angular resolution, dynamical measurements of the gas and stars at the center of the Milky Way suggested the presence of $\sim 3 \times 10^{6} M_{\odot}$ of dark matter and confined it to within a radius of $\sim 0.1 \mathrm{pc}$. The implied minimum dark matter density of $\sim 3 \times 10^{9} M_{\odot} p c^{-3}$, however, still allowed a cluster of dark objects, such as neutron stars 
or stellar mass black holes, as one of the alternatives to a single supermassive black hole, because the measurements did not force the cluster's lifetime to be shorter than the age of the Galaxy (Maoz et al. 1998).

Significant progress has been made recently with diffraction-limited near-infrared studies of the central stellar cluster. The first phase of these experiments yielded proper motion velocities (Eckart \& Genzel 1997; Ghez et al. 1998), which suggested that $2.6( \pm 0.6) \times 10^{6} M_{\odot}$ of dark matter is confined to within $0.015 \mathrm{pc}$. This increased the implied minimum dark matter density by 3 orders of magnitude to $10^{12} M_{\odot} p c^{-3}$ and eliminated a cluster of dark objects as a possible explanation of the Galaxy's central dark mass concentration (Maoz et al. 1998), but still left the fermion ball hypothesis (e.g., Tsiklauri \& Viollier 1998; Munyaneza \& Viollier 2002) as an alternative to a single supermassive black hole. The velocity dispersion measurements also localized the dark matter's centroid to within 100 mas and at a position consistent with the nominal location of the unusual radio source Sgr A* (Ghez et al. 1998), whose emission is posited to arise from accretion onto a central supermassive black hole (e.g., Lo et al. 1985). The detection of acceleration for three stars - S0-1, S0-2, and S0-4 localized the dark mass to within 30 mas, increased the dark matter's minimum density to $10^{13} M_{\odot} p c^{-3}$, and thereby further strengthened both the case for a supermassive black hole and its association with Sgr A* (Ghez et al. 2000; Eckart et al. 2002).

Deviations from linear motions also initiated a new phase for these proper motion experiments, that of direct orbital studies. By making a number of assumptions, including fixing the central mass to the value obtained from the velocity dispersion analysis and its location to that inferred for Sgr A* by Menten et al. (1997), Ghez et al. (2000) and Eckart et al. (2002) obtained the first crude orbital solutions; these experiments revealed that orbital periods for S0-2 and S0-1 could be as short as 15 and 35 years, respectively. With a larger fraction of the orbit being traced, more precise orbital analyses have been carried out for S0-2 by Schödel et al. (2002), who dropped the mass assumption, and by Ghez et al. (2003), who dropped both the mass and center of attraction assumptions and added radial velocity measurements. These orbital solutions suggested that S0-2 made a closest approach of 0.0006 pc (120 AU) in 2002 and that its orbit encloses a central mass of $3.7( \pm 1.5) \times 10^{6} M_{\odot}$ (Schödel et al. 2002) or $4.0( \pm 0.6) \times 10^{6} M_{\odot}\left(\right.$ Ghez et al. 2003) ${ }^{1}$, which is somewhat higher than that found from the velocity dispersion measurements. Possible causes of this discrepancy include (1) inaccuracies in the assumptions made in the use of the velocity-dispersion-based

\footnotetext{
${ }^{1}$ The uncertainties in the estimated mass in Ghez et al. (2003) are a factor of 2.5 smaller than that in Schödel et al. (2002), despite the two additional free model parameters introduced by fitting for the center of attraction and the shorter time baseline. This is primarily due to the higher astrometric accuracy of the Keck data set, rather than the inclusion of radial velocity measurements.
} 
projected mass estimators about the stellar cluster's number density distribution and/or the level of anisotropy (Genzel et al. 2000; Figer et al. 2003), (2) inaccuracies in the orbital fit assumptions, such as the central mass distribution being point-like and at rest with respect to the central stellar cluster, and (3) systematic errors in either the overall velocity dispersion measurement for the central stellar cluster or S0-2's individual positional measurements. Further measurements are necessary to determine whether this $2 \sigma$ difference in the estimates of the central dark mass produced by the two methods is real and, if so, what its origin is.

While the detection of spectral lines in S0-2 provided full dynamical information, it also offered insight, for the first time, into the nature of this star that is orbiting in such close proximity to the central dark mass. S0-2's spectral features are consistent with those of an O8-B0 dwarf, suggesting that it is a massive $\left(\sim 15 M_{\odot}\right)$, young $(<10 \mathrm{Myr})$, main sequence star (Ghez et al. 2003). Less direct measurements of other stars within the central $1^{\prime \prime} \times 1^{\prime \prime}$, which are known collectively as the Sgr A* stellar cluster, imply that these stars might be similarly young; specifically, their similar $2 \mu \mathrm{m}$ luminosities and the lack of CO absorption in spectra of individual stars (Genzel et al. 1997; Gezari et al. 2002) or in integrated spectra of the Sgr A* stellar cluster (Eckart, Ott, \& Genzel 1999; Figer et al. 2000) lead to the conclusion that they, like S0-2, have hot photospheres consistent with massive young stars.

While the presence of young stars in close proximity to our Galaxy's supermassive black hole has long been recognized as a problem in the context of the young He I emission-line stars (Sanders 1992; Morris 1993), this problem is much worse for the Sgr A* cluster stars, whose distances from the black hole are an order of magnitude smaller. At S0-2's apoapse distance of $0.01 \mathrm{pc}$, inferred from the orbital solutions, the Roche density is $10^{14} \mathrm{~cm}^{-3}$, whereas the maximum density determined for even the nearby circumnuclear disk, located at radii of $\sim 1-3 \mathrm{pc}$, is only about $10^{5}-10^{6} \mathrm{~cm}^{-3}$ (e.g., Jackson et al. 1993; Christopher \& Scoville 2003). Furthermore, at present, the region over which S0-2 is currently orbiting contains only a very low-density plasma, as evidenced by weak Br- $\gamma$ line emission (Figer et al. 2000; Gezari et al. 2002). Several ideas proposed to account for the apparently young He I emission-line stars may be applicable to the Sgr A* cluster stars and they fall into the following three broad categories: (1) the stars are indeed young and formed in-situ, which requires much higher local gas densities in the recent past in order to enable star formation to proceed in the black hole's strong tidal field (e.g., Levin \& Beloborodov 2003), (2) the stars are young and formed at larger radii, where the black hole's tidal effects are small, and underwent rapid orbital migration inwards (e.g. Gerhard 2001; Kim \& Morris 2003; Hansen \& Miloslavjevic 2003), and (3) the stars are old (initially formed long ago), but their appearance has been altered, due to interactions with the local environment, such that they appear young but have had sufficient time to migrate inwards from their original birth place 
(e.g., Morris 1993; Genzel et al. 2003a). Stellar kinematics produced by these mechanisms are likely to differ. Well constrained orbits for a set of stars in the Sgr A* cluster would allow a direct examination of the cluster's kinematics and therefore would provide important insight into how these stars formed and came to be on their present orbits.

This paper reports new proper motion measurements obtained with the W. M. Keck 10-meter telescope for 4 previously known stars (S0-1, S0-2, S0-4, S0-5) and for 3 newly identified stars (S0-16, S0-19, S0-20). The trajectories of all of these stars show significant curvature or linear acceleration, thus allowing the first simultaneous orbital analysis for multiple stars making their closest approaches to the central dark mass. Section 2 describes the observations, which now cover an 8-year time baseline. Section 3 provides the details and results for source identification, astrometry, and the orbital fits, which, for the first time, allow for the dark mass' motion on the plane of the sky. Section 4.1 discusses the constraints that the orbital parameters offer on the nature of the central dark mass distribution, which has become the best case yet for a supermassive black hole at the center of any normal type galaxy and whose mass, position and motion in the infrared reference frame are determined with unprecedented accuracy. Finally, Section 4.2 explores how the direct measurements of orbital dynamics impact the question of the origin of the central stellar cluster.

\section{Observations}

New $K[2.2 \mu m]$-band speckle imaging observations of the Galaxy's central stellar cluster were obtained with the W. M Keck I 10-meter telescope using the facility near-infrared camera, NIRC (Matthews \& Soifer 1994; Matthews et al. 1996) on the nights of 2000 April 21, 2000 May 19-20, 2000 July 19-20, 2000 Oct 18, 2001 May 7-9, 2001 July 28-29, 2002 April 23-24, 2002 May 23-24 \& 28-29, 2002 June 2, 2002 July 19-20, 2003 April 21-22, 2003 July 22-23, and 2003 September 7-8. These data sets were collected and analyzed similarly to the data sets obtained between 1995 and 1999 for this project (see Ghez et al. 1998, 2000 for details). In summary, short $\left(t_{\exp }=0.1 \mathrm{sec}\right)$ exposures were obtained in sets of $\sim 200$, resulting in a total of $\sim 7,000$ exposures per observing run. Each frame, with a scale of $20.396 \pm 0.042$ mas pixel $^{-1}$ (see Appendix B) and a corresponding field of view of 5." $22 \mathrm{x}$ 5."22, was sky-subtracted, flat fielded, bad-pixel-corrected, corrected for distortion effects, and magnified by a factor of two. In sets of 200, the frames were shifted to the location of the brightest speckle of IRS $16 \mathrm{C}(\mathrm{K}=9.8 \mathrm{mag})$ and combined to create intermediate shiftand-add (SAA) maps, which have point spread functions (PSF) that can be described as containing a diffraction-limited core on top of a seeing halo. These were then combined after applying a seeing cut, which required that the seeing halo FWHM be less than $\sim-0 .{ }^{\prime \prime} 4-0 . " 6$, 
depending on the overall quality of the night. Final SAA maps have point spread functions composed of a diffraction-limited core $\left(\theta \sim 0 .{ }^{\prime \prime} 05\right)$, containing $\sim 4 \%$ of the radiation, on top of a halo that has a FWHM of $\sim 0 . " 4$. In addition to averaging all the data from each run to produce a final SAA map, these data were divided into three sub-sets to construct "sub-maps", which were used to determine positional and brightness uncertainties.

\section{Data Analysis \& Results}

\subsection{Source Identification}

Sources are identified using the same procedure described by Ghez et al. (1998), with a few minor modifications. As in Ghez et al. (1998), a "match filter" is applied to each image, by cross-correlating the image with the core of its PSF, out to a radius of 0."06 (see Figure 1). In a first pass at source identification, correlation peaks larger than a threshold value are flagged as stars. Once stars are identified, a second lower threshold value is used to track these stars in images in which they were not identified with the first threshold value; this second pass search is limited to within a specified radius of the predicted position. Positions of sources found in either the first or second pass search are estimated based on the correlation map peak and only sources that are identified in at least 3 epochs are included in our final proper motion sample. While in Ghez et al. (1998) the predicted position for the second pass source search was simply the position found in the first pass, here we use any kinematic information available from the first pass to define this predicted position. Two other modifications change only the values used in the algorithm. We lowered the first pass threshold correlation value for source identification from 0.7 to 0.5 , which allows fainter sources to be identified, and we have decreased the second pass search area radius from $0^{\prime \prime} 07$ to the uncertainty in the predicted position (with the constraint that it must be at least $0{ }^{\prime \prime} 01$ and no more than $0{ }^{\prime} .07$ ), due to the increased number of sources that are being tracked. Positions are now estimated using gaussian fits, as opposed to a simple centroiding algorithm. The final modification requires that each source be detected in all three sub-maps (see $\S 2$ ); first and second pass sources had sub-map correlation thresholds of 0.3 and 0.2 , respectively.

Photometric values are estimated using two methods. First, simple aperture photometry, as described in Ghez et al. (1998), is applied to help track the sources through the data set. Second, PSF fitting with StarFinder (Diolaiti et al. 2000) is implemented and these are the values (average and $\mathrm{rms}$ ) reported in Table 1. StarFinder and aperture photometry produce the same results for bright $(K \lesssim 15.2)$ sources, but, for the fainter sources, the StarFinder results are somewhat fainter $(\sim 0.2 \mathrm{mag})$ and more precise, due to the stellar 
confusion. In this study, the zero points are established based on Blum et al. (1996) measurements of IRS $16 \mathrm{C}(\mathrm{K}=9.83 \mathrm{mag})$, IRS 16NW (K=10.03 mag), and IRS 16NE (K=9.00 $\mathrm{mag}$ ), which results in magnitudes that are $\sim 0.2 \mathrm{mag}$ fainter than than those reported in Ghez et al. 1998, which relied on IRS16NE only for a zero point estimate.

While many sources are identified and tracked over our entire $\sim 5^{\prime \prime} \times 5^{\prime \prime}$ field of view, this study is limited to sources within a radius of 0.4 of the infrared position for Sgr A* (see Appendix B); the radius is set by the criterion that all stars with accelerations of 2 mas $\mathrm{y}^{-2}$ or greater should reside within this region, assuming a mass, $M$, of $3.7 \times 10^{6} M_{\odot}$ (see $\S 4.1$ ), or equivalently $r_{\max }^{2}=G M / a_{\min }$.

This procedure identifies 17 proper motion sources $(K \lesssim 16.8)$, of which 10 are newly discovered in this study ${ }^{2}$ and all of which are shown in Figure 1. The new sources are fainter than the sources in this study that were previously published $\left(K_{\text {new }} \gtrsim 15.1 \mathrm{mag}\right)$, with only one exception (S0-8, which is located at the largest projected separation). Among the original proper motion sample reported in Ghez et al. (1998), there are many other sources comparably faint to the newly discovered proper motion sources, but at larger radii; the reason for this is that at the center of the maps source confusion lowers the correlation values and reduces the sensitivity to faint sources using our source identification technique. The new source detections are therefore a consequence of our lower correlation thresholds and, as can be seen in Figure 1, these thresholds are still fairly conservative, since a number of additional sources are seen in the cross-correlation maps. While the previously known sources are detected in all the maps, the new sources are not, due to the variation in the maps' sensitivities and, occasionally, confusion with a brighter source (see footnotes in Table 1). Nonetheless, sources as faint as $\sim 16.7$ are detected in the majority of maps in the second pass for source detections and sources brighter than $\sim 15.5$ mag are detected in all maps in the first pass. Table 1 lists the properties of all the detected sources in our sample; the new sources are named according to the convention introduced in Ghez et al. 1998 and summarized in Appendix A.

\subsection{Astrometry}

Stellar astrometry is derived in three separate steps. First, centroid positions on the correlation peaks provide estimates of the stars' locations in each of the maps. Uncertainties in these locations are estimated based on the rms of their locations in the 3 sub-maps created

\footnotetext{
${ }^{2}$ We note that after this paper was submitted for publication, 6 of the 10 new sources were also reported by Schödel et al. (2003).
} 
for each map (see $\S 2$ ) and have an unweighted average value of $\sim 2$ mas for the brightest stars $(\mathrm{K}<15 \mathrm{mag}$ ) and $\sim 5$ mas for the $\mathrm{K} \sim 15.5$ - $15.7 \mathrm{mag}$ stars. Second, the coordinate system for each map is transformed, with the application of a net translation and rotation, to a common local reference frame. As in Ghez et al. (1998), this transformation is determined by minimizing the net motion of the measured stars, but with the three modifications: (1) rather than using all stars detected in the central $\sim 5^{\prime \prime} \times 5^{\prime \prime}$, we now exclude those stars within 0 .'5 of $\operatorname{Sgr} A^{*}$ as well as those that have correlation values less than $0.7,(2)$ the common local reference frame is now chosen to be the map obtained at the middle epoch, 1999 July, instead of the 1995 June map, in order to minimize the uncertainty in the coordinate transformations, which increase with the temporal distance from the reference epoch, and (3) a second pass through the minimization process is applied using initial estimates of the proper motions from the first pass. As described in Ghez et al. (2000), positional uncertainties associated with this transformation of the relative positions of stars into a common coordinate system are estimated by a half-sample bootstrap method. These uncertainties are minimized at the center of the field of view, which is where the stars reported here were always observed, and that decrease with both the number of stars included in estimating the transformation (48 - 104 stars) and the closer in time the epoch is to the reference epoch. The values of these uncertainties for the stars in this study range from zero, for the reference epoch of 1999 July, to 0.44, for epochs close to 1999 July, and up to 2.5 mas, for data sets at the extrema in time of our experiment. Compared to the centroiding uncertainties, the transformation uncertainties are negligible for the faintest stars and are, in some epochs, comparable to that of the brightest stars in our sample. The uncertainties from the centroiding and transformation processes are added in quadrature to produce the final relative position uncertainties. It is these relative positions that are used in the orbital analysis presented in $\S 3.3 .1$ and their weighted averages are presented in column 10 of Table 1. The third and final aspect of the astrometric measurements is transforming the relative positions and orbital solutions from the 1999 July map's coordinate system to an absolute coordinate system using measurements of sources with known absolute astrometry, as described in Appendix B; this final transformation has been applied to the orbital solutions presented in §3.3.2.

Stellar confusion not only prevents faint sources from being detected (as discussed above), but can also generate astrometric biases. Fortunately, the sources' high proper motions easily reveal the underlying biasing sources at later or earlier times, so that these biases can be recognized. We exclude the biased points in a three step procedure. First, measurements of stars during epochs in which they are obscuring other stars are temporarily excluded; at this point, stars with remaining measurements in less than three years (S0-3 and S0-21) are removed from further dynamical analysis due to our inability to assess the possible effects of astrometric biases. Second, with this vetted data set, we carry out preliminary 
linear or orbit model fits, in which no parameters are constrained (see §3.3.1). Third, we return to the original data set and remove those points that are offset by more than $3 \sigma$ from the preliminary best fit model. Column 5 of Table 1 summarizes the number of remaining points that are used in the fits described in $§ 3.3 .2$.

\subsection{Orbital Analysis}

Significant curvature or linear acceleration in the plane of the sky is detected for 7 of the 17 sources listed in Table 1, using the criterion defined by Ghez et al. (2000), but accounting for the differing number of epochs by considering $\chi_{\text {tot }}^{2}$ instead of $\chi_{\text {dof }}^{2}$ (see column 11 in Table 1). Stars are considered to show significant deviations from linear proper motion, if they have $\Delta \chi_{t o t}^{2}$, which is the difference between the total $\chi^{2}$ value resulting from the best linear fit and the total $\chi^{2}$ from the best second order polynomial fit, greater than 15. S0-1, S0-2, S0-4, S0-5, S0-16, S0-19, and, S0-20, satisfy this criterion and have accelerations in the plane of the sky of at least 2 mas $\mathrm{y}^{-2}$ and as much as 1500 mas $\mathrm{y}^{-2}$ and, of these, S0-2, S0-16, and S0-19 have measurable higher order positional derivatives. We therefore carry out orbital fits for these 7 stars with a model described in $§ 3.3 .1$ and with resulting orbital parameters given in $\S 3.3 .2$.

\subsubsection{Model \&3 Method}

We assume a model in which the gravitational potential arises from a single dominant point mass, which allows multiple stars to contribute simultaneously to the solution for the following properties of the central object:

- Mass $(M)$

- Location $\left(r_{R A}, r_{D E C}\right)$

- Linear motion on the plane of the sky $\left(v_{R A}, v_{D E C}\right)$.

In this analysis, the point source's distance $\left(R_{o}\right)$ and its linear motion along the line of sight $\left(v_{z}\right)$ are not solved for; to get $M, R_{o}$ is assumed to be $8 \mathrm{kpc}$ (Reid 1993), while $v_{z}$ is set to 0 . Setting $\mathrm{v}_{z}$ equal to $0 \mathrm{~km} \mathrm{~s}^{-1}$ is reasonable given that the resulting limits on the values of $\mathrm{v}_{R A}$ and $\mathrm{v}_{D E C}\left(\lesssim 76 \mathrm{~km} \mathrm{~s}^{-1}\right)$ are comparable to the uncertainties on the radial velocity measurements for S0-2 ( $\sim 40 \mathrm{~km} \mathrm{~s}^{-1}$, Ghez et al. 2003), which are the only radial velocities used in this analysis; furthermore, Figer et al. (2003) find an average $\mathrm{v}_{z}$ for a set of cool 
stars at the Galactic Center consistent with 0 to within $11 \mathrm{~km} \mathrm{~s}^{-1}$. In addition to these 5 common free parameters, there are the following 6 free parameters for each star:

- Period $(P)$, which, when combined with the estimate of the central dark mass, yields the angular semi-major axis $(A)$

- Eccentricity $(e)$

- Time of periapse passage $\left(T_{o}\right)$, which is when the star comes closest to the central dark mass

- Inclination (i), which is the angle between the normal to the orbital plane and the line of sight and has values ranging from 0 to $180^{\circ}$, with values less than $90^{\circ}$ corresponding to direct motion (position angles increasing with time) and values greater than $90^{\circ}$ corresponding to retrograde motion,

- Position angle of the nodal point $(\Omega)$, which is the position angle, measured Eastward of North, of the line of intersection between the plane of the sky through the central dark mass and the orbital plane. In the absence of radial velocity measurements (e.g., all stars except S0-2), it is not possible to distinguish between the ascending and descending nodes, which correspond to the nodal points where the star is moving away from and towards us, respectively, and, by convention, the value less than $180^{\circ}$ is taken; this ambiguity generates a similar $180^{\circ}$ ambiguity in the longitude of periapse. With radial velocity measurements (e.g., S0-2, Ghez et al. 2003; Eisenhauer et al. 2004), these ambiguities are removed and the ascending node is given for $\Omega$, with permitted values ranging from 0 to $360^{\circ}$.

- Longitude of periapse $(\omega)$, which is the angle in the plane of the orbit, in the direction of motion, from node to periastron, with permitted values ranging from 0 to $360^{\circ}$.

In total, this model contains $5+N \times 6$ parameters, where $N$ is the number of stars included in the simultaneous fit. This is a more powerful approach than simply averaging the results of $\mathrm{N}$ independent orbital analyses, since each star in a simultaneous solution contributes to the determination of the common parameters, which in turn leads to a better definition of each star's orbital parameters.

The orbital fits, shown in Figure 2, are carried out by minimizing the chi-squared value between the data and the model and the reported uncertainties are obtained from the covariance matrix, which corresponds roughly to changing the total chi-squared values by 1 . In total, the data set consists of 254 measurements - 126 positional measurements, each of 
which provides two independent data points (one for the East-West position and the other for the North-South position), and 2 radial velocity measurements of S0-2 from a single year, reported by Ghez et al. (2003). While the final orbital parameters reported in Tables 2 \& 3 come from a simultaneous fit, which is described in detail below, we first carry out a number of independent and semi-independent orbital solutions to check the validity of using common values for the central dark mass as well as to check our estimates of the positional uncertainties. In these preliminary fits, the central dark mass is not allowed to move and we scale all the estimated relative position uncertainties by a scale factor, which produces a $\chi_{\text {dof }}^{2}$ of 1 for the best independent fits; these scale factors modify the astrometric uncertainties by at most only $30 \%$ and on average by only $10 \%$. The fully independent solutions yield locations for the central dark mass that are consistent to within $2 \sigma$, with individual uncertainties of $\sim 1,4$, and 25 mas for S0-2, S0-16 and S0-19, respectively, and larger uncertainties for the remaining stars. Consistency for the central dark mass is checked by carrying out a semiindependent fit in which the central dark object's location is treated as a common parameter, but its mass is not. This fit is carried out with the 3 stars - S0-2, S0-16, and S0-19 - that yield meaningful independent mass estimates $(M / \delta M>3)$, which are consistent to within $2 \sigma$, with uncertainties of $0.2,0.6,1.5\left(\times 10^{6}\right) \mathrm{M}_{\odot}$, respectively. It therefore appears to be well justified to simultaneously fit the data with a model in which the central dark object's properties $\left(\mathrm{M}, r_{R A}, r_{D E C}, \mathrm{v}_{R A}\right.$, and $\left.\mathrm{v}_{D E C}\right)$ are common to all the stars. Using an algorithm described by Salim \& Gould (1999), we solve for the orbital parameters simultaneously with the inclusion of the central dark object's linear motion on the plane of the sky as a free parameter. Since S0-2, S0-16, and S0-19 are the only stars that have any significant implications for the central dark object's properties, we divide the problem into two. A three-star simultaneous fit with $\mathrm{S} 0-2$, S0-16, and S0-19 provides the orbital parameters for these three stars as well as the central dark objects properties. The orbital parameters for each of the remaining stars are obtained from a four-star simultaneous fit, which includes the star in question plus S0-2, S0-16, and S0-19; this was done to appropriately include the effects of the uncertainties in the central dark object's parameters in estimates of the remaining stars' orbital parameters. The resulting $\chi_{d o f}^{2}$ for all the simultaneous fits are comparable to 1 , again supporting the use of a point mass potential model.

\subsubsection{Orbital Fit Results}

Estimates of the central dark mass' properties from the three-star simultaneous fit are reported in Table 2. The central dark mass is estimated to be $3.7( \pm 0.2) \times 10^{6}\left(\frac{R_{o}}{8 k p c}\right)^{3} M_{\odot}$. While this is consistent with that inferred from the orbit of S0-2 alone (Ghez et al. 2003; Schödel et al. 2003), its uncertainty is a factor of 3-4 times smaller due, primarily, to the 
longer time baseline for the measurements, and, in part, to the additional information offered by S0-16 and S0-19. This makes distance, which is fixed in all the orbital analyses reported thus far, the limiting uncertainty for the first time (see also Eisenhauer et al. 2004); the $0.5 \mathrm{kpc}$ uncertainty in the Galactic center distance (Reid 1993) contributes an additional $19 \%$ uncertainty in the estimated mass, beyond that reported in Table 2. Similarly to the mass, the inferred center of attraction agrees well with the results from the analysis of S0-2's orbit by Ghez et al. (2003). The location is only modestly improved in the simultaneous fit, because the black hole's proper motion is treated as an unknown variable only in the multiple star orbit model, which increases the formal uncertainties in the black hole's location. The estimate of the dark mass's motion on the plane of the sky is the first such estimate derived from orbital fits. While a single star's orbital trajectory can, in principle, constrain this motion, in this solution it is primarily constrained by the closest approaches of S0-2, S0-16, and S0-19 and their span of periapse passage times of 5 years. The inferred proper motion of

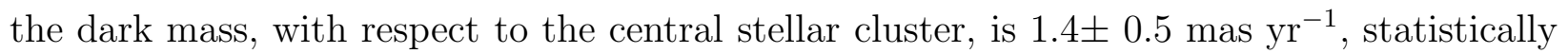
consistent with no motion. Overall, simultaneously fitting the stellar orbital motion has allowed significant improvements in the derivation of the central dark object's properties.

With the central parameters constrained simultaneously by multiple stars, the precision with which each star's orbital elements can be determined is also greatly improved compared to that obtained from an independent orbit analysis. Table 3 lists the parameters specific to the individual stars from the simultaneous fit. Over the course of this study (1995 - 2003), these stars have either undergone periapse passage or are remarkably close to periapse. The smallest periapse distance is achieved by $\mathrm{S} 0-16$, which comes within $45 \mathrm{AU}$ with a velocity of $12,000 \pm 2,000 \mathrm{~km} \mathrm{~s}^{-1}$.

There are clear selection effects in this study that must be understood and accounted for before the ensemble properties of the sample can be studied. Since a star has to experience

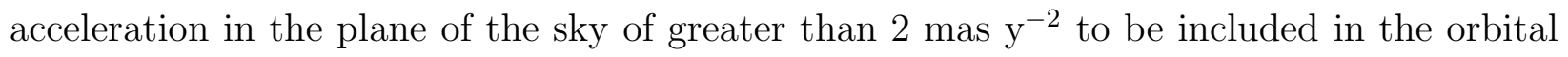
analysis, there is an observational bias towards detecting stars in eccentric orbits at periapse, in spite of the fact that a star spends most of its time away from periapse. Stars experience their largest acceleration near periapse, at a projected distance which scales as $q=A(1-e)$. For a given semi-major axis above $\sim 3200 \mathrm{AU}$, this allows stars in highly-eccentric orbits to have detectable accelerations near their closest approach, while stars on low-eccentricity orbits will be below the detection threshold in all parts of their orbits. Figure 3 quantifies these effects based on the fraction of time a face-on orbit experiences accelerations larger than our threshold value. Four stars - S0-2, S0-16, S0-19, and S0-20 - lie in the parameter space that is unbiased, $1 \mathrm{star}-\mathrm{S} 0-1$ - resides in a region that is mildly biased ( $~ 50 \%$ effect), and the last 2 stars - S0-4 and S0-5 - are detected only because they are on eccentric orbits and remarkably close to periapse passage. The excess of high eccentricity orbits in our sample 
is therefore a consequence of an observational bias; restricting the analysis to the 5 stars for which the bias is $\lesssim 50 \%$ effect, we find 2 stars with eccentricities $\lesssim 0.70$, which is statistically consistent with isotropy, if we assume that an isotropic system has a cumulative probability distribution $\propto e^{2}$ (Binney \& Tremaine 1987).

The distribution of semi-major axes is also noteworthy. While there are no observational selection effects against it, there is a distinct lack of stars as bright as those tracked in this study ( $\mathrm{K} \lesssim 15.5$ ) having semi-major axes $\lesssim 1000$ AU, and likewise apoapse distances of $\lesssim 1800$ AU. The other end of these distributions, however, are not seen due to selection effects.

In contrast to the shape of the orbit, its orientation should be unaffected by observational bias. To fully describe the orientations of the orbits, it is necessary to specify the directions of two vectors, one normal to the orbital plane, such as the angular momentum vector, and one along the semi-major axis, such as the direction to apoapse. For S0-2, which has both astrometric and radial velocity measurements, the full three-dimensional orbit is unambiguously determined. For the stars with only astrometric measurements available, however, a degeneracy exists from a possible reflection about the plane of the sky; we therefore assume that these orbits are oriented such that the unit angular momentum and apoapse direction vectors are in the same hemisphere as those quantities derived for S0-2. Figures 4 and 5 show the directions for these unit vectors. The directions of neither the angular momentum nor apoapse vectors show any clear preferred direction or co-planarity, and are statistically consistent with a random distribution of orbits.

\section{Discussion}

\subsection{The Case for and Properties of the Central Supermassive Black Hole}

Stellar orbits provide the most direct measure of the amount of dark matter concentrated at the center of the Galaxy. Compared to masses inferred from the velocity dispersion measurements, the mass derived from multiple orbits, $3.7( \pm 0.2) \times 10^{6} M_{\odot}$, is a factor of 2 higher than that estimated by a non-parametric approach presented by Chakrabarty \& Saha (2001), which is supposed to be the most robust approach, and is somewhat less discrepant with the parametric approaches (e.g., Ghez et al. 1998; Genzel et al. 2000; Schödel et al. 2003). Since the mass estimates from the velocity dispersion measurements and orbital fits have all assumed the same distance and all depend on distance as $R_{o}{ }^{3}$, the assumptions about distance are not the source of this mass discrepancy. By simultaneously solving for multiple orbits, we now have only one more parameter left out of the fit for a Keplerian orbit model, that of the black hole's motion along the line of sight. Given the small values 
for its motion on the plane of the sky, this last parameter is unlikely to have any significant effect on the estimated mass. The two possibilities therefore lie in problems with the mass estimates from the velocity dispersions. First, the mass estimates could be biased by the weighting schemes used to calculate the velocity dispersions. While roughly 100 stars have reported proper motion values in the earlier works of Ghez et al. 1998 and Genzel et al. 2000 , only 18 of these have $\mathrm{S} / \mathrm{N}>5$ and half of them have $\mathrm{S} / \mathrm{N}<3$, making the velocity dispersion bias term non-negligible. Second, the projected mass estimators could be biased by the properties of the central stellar cluster. Specifically, both the level of anisotropy and the slope of the stellar density distribution can significantly alter the values inferred from standard projected mass estimators (Genzel et al. 2000; Figer et al. 2003). Different results have been reported both for the presence of anisotropy (Ghez et al. 1998; Genzel et al. 2000; Schödel et al. 2003) and the radial distribution of stars (Scoville et al. 2003; Genzel et al. 2003a; Figer et al.). While a full exploration of these effects is outside the scope of this paper, here we emphasize that the orbital mass is more robust and should be used in all future characterizations of the Galaxy's central dark mass concentration.

Stellar orbits confine the central dark mass of $3.7( \pm 0.2) \times 10^{6}\left(\frac{R_{o}}{8 k p c}\right)^{3} M_{\odot}$ to within $45 \mathrm{AU}$, the closest approach of S0-16, implying a minimum density of $8 \times 10^{16} M_{\odot} / p c^{3}$ for the central dark mass. This confines the mass to a volume that is a factor of 20 smaller than that inferred from S0-2 (Schödel et al. 2002; Ghez et al. 2003) and increases the inferred density by four orders of magnitude compared to estimates from measurements of acceleration vectors (Ghez et al. 2000; Eckart et al. 2002). At this density, the two existing alternative explanations to a supermassive black hole for the compact dark object found at the center of the Galaxy become significantly less tenable (see also Schödel et al. 2002). Any cluster of dark objects, such as those considered by Maoz (1998), would have a lifetime of a mere $\sim 10^{5}$ years, owing to gravitational instability, which is significantly shorter than the age of the Galaxy, making this a highly unlikely explanation for the central dark mass concentration. For the fermion ball hypothesis (Viollier et al. 1993), the mass of the constituent particles is now required to be $74 k e V c^{-2}\left(\frac{0.3}{R}\right)^{3 / 8}\left(\frac{2}{g}\right)^{1 / 4}\left(\frac{3.7 \times 10^{6}}{M}\right)^{1 / 8}$, where $\mathrm{R}$ and $\mathrm{M}$ are the radius in milli-pc and mass in $\mathrm{M}_{\odot}$ of the fermion ball, respectively, and $\mathrm{g}$ is the spin degeneracy factor of the fermion; this is 5 orders of magnitude larger than the current limits on degenerate neutrino species (Spergel et al. 2003), rendering the fermion ball hypothesis also highly unlikely (see also discussion in Schödel et al. 2002). With the Galaxy's central dark mass now confined to a radius equivalent to $600 \times$ the Schwarzchild radius of a $3.7 \times 10^{6} M_{\odot}$ black hole, the multiple stellar orbits present the strongest case yet for a supermassive black hole at the center of the Milky Way galaxy.

The measured linear velocity of the central black hole on the plane of the sky limits the mass of any possible companion black hole, through the assumption that any velocity 
is due to reflex motion. With a $1 \sigma$ upper limit of 2 mas $^{-1}$, the mass of any possible companion black hole is constrained to be less than $\sim 5 \times 10^{5}(R / 16,000 A U)^{1 / 2} M_{\odot}$, where $R$ is the distance of the companion black hole from the central black hole; the generalization of this limit to other radii works as long as the black hole companion lies outside the orbits that contribute to the determination of the central dark object's properties (Table 3) and that the orbital period is long compared to the duration of the study, 8 years. A related measurement comes from upper limits inferred for the motion of the radio source Sgr A*, which is assumed to be associated with the central black hole (see discussion in §4.2). In the plane of the Galaxy, the upper limit is $\sim 20-25 \mathrm{~km} \mathrm{~s}^{-1}$ (Backer \& Sramek 1999; Reid et al. 1999; Reid \& Brunthaler 2004, which is comparable to our limits. Perpendicular to the Galaxy, Reid \& Brunthaler (2004) derive a more constraining upper limit of $\sim 1 \mathrm{~km}$ $\mathrm{s}^{-1}$. Nonetheless, the infrared and radio measurements are fundamentally different. In the infrared, the black hole's motion is measured with respect to the central stellar cluster, which traces the local barycenter, while in the radio, Sgr A*'s motion is derived with respect to background quasars, so motions of the black hole along with the central stellar cluster as well as the solar parallax show up in this measurement. Therefore, while the radio upper limit on the motion of Sgr A* is smaller than the infrared upper limit on the motion of the black hole, the latter is a more direct measure of the upper limit for the reflex motion from a possible black hole companion.

In the context of other galaxies, the Milky Way's central dark mass concentration distinguishes itself in terms of both its inferred density and mass. The Galaxy's central minimum dark mass density now exceeds the minimum dark matter density inferred for NGC 4258 (Greenhill et al. 1995; Miyoshi et al. 1995) by five orders of magnitude, reinforcing the Milky Way as the strongest case for a black hole at the center of any normal type galaxy.

It is also possible to use the observed dark mass concentration in the Milky Way to further explore the fermion ball hypothesis as a universal alternative explanation for supermassive compact objects in all galaxies as has been proposed in the past. For objects composed of the minimum mass particles imposed by the stellar orbits in the Galactic center, the maximum mass is $1 \times 10^{8} M_{\odot}\left(\frac{76 \mathrm{keV}}{m c^{2}}\right)^{2}\left(\frac{2}{g}\right)^{1 / 2}$, from the Oppenheimer-Volkoff limit (Munyaneza \& Viollier 2002). This is less massive than half of the supermassive compact objects that have been identified thus far (cf., for example, the compilation in Tremaine et al. 2002), thereby eliminating an all-encompassing fermion ball hypothesis.

In contrast to its high minimum central dark mass density, the Milky Way appears to harbor the least known massive supermassive black hole, as inferred directly from dynamical measurements. It therefore potentially has an important role to play in assessing the $\mathrm{M}_{b h}$ vs. $\sigma$ relations (e.g., Ferrarese \& Merritt 2000; Gebhardt et al. 2000). However, the current 
impact of the Milky Way on the $\mathrm{M}_{b h}$ vs. $\sigma$ relation is limited by uncertainties in the determination of its bulge velocity dispersion (Tremaine et al. 2002). Nonetheless, the higher mass value from the orbits brings our Galaxy into better agreement with the $\mathrm{M}_{b h}$ vs. $\sigma$ relationship derived from a large sample of galaxies (e.g., Tremaine et al. 2002; Merritt \& Ferrarese 2001).

\subsection{Sgr $A^{*}$ and other Possible Counterparts to the Central Black Hole}

The orbits provide very precise information on the location and kinematics of the central supermassive black hole, allowing us to explore its association with the radio source Sgr A* and any possible near-infrared counterparts. In Appendix B, we derive the infrared position of the radio source Sgr A*. Relative to the dynamically determined position of the central dark mass, which is known to within 1.3 mas $(1 \sigma)$, the inferred infrared position of Sgr A*, which is less accurately known - is offset by a mere $0.5 \pm 6.4$ mas $\mathrm{W}$; and $9 \pm 14$ mas $\mathrm{S}$; the two positions therefore appear to be consistent to within $1 \sigma$. Furthermore, using the kinematics of S0-2 from Ghez et al. (2003) and the upper limit on the motion of Sgr A*, Reid et al. (2004) argue that Sgr A* has a minimum mass of $4 \times 10^{5} M_{\odot}$, consistent with the black hole mass estimated from orbital motion. Given the agreement in position, velocity (discussed in $\S 4.1$ ), and mass, it appears that $\mathrm{Sgr} \mathrm{A}^{*}$ is indeed associated with the black hole at the Galaxy's center.

Identifying near-infrared counterparts to the central black hole is a difficult task, given the high stellar densities, velocities, and accelerations at that location. S0-19 serves as a good illustration of these challenges. Its large proper motion and strong curvature in a crowded region makes it challenging to track and led Genzel et al. (1997) to propose their 1996.43 detection of this source (their label S12) as the best candidate for the infrared emission from the central black hole; at that time, this source was coincident to within $1 \sigma$ (30 mas) of the relatively crude position of Sgr A* reported by Menten et al. (1997). With the newly determined location of the black hole based on orbits, it is now clear that this source is offset by 54 mas, or $41 \sigma$, from $\operatorname{Sgr} \mathrm{A}^{*}$ and that it is simply one data point in the trajectory of the high velocity star S0-19 that, in 1996, was near the black hole ${ }^{3}$.

The search for infrared counterparts to the central black hole is greatly facilitated by the

\footnotetext{
${ }^{3}$ S0-19 was detected by Ghez et al. (1998) in 1995 with two possible counterparts identified in 1996. With limited time coverage, Ghez et al. (1998) were not able to definitively identify either as the correct counterpart to either S0-19's 1995 position or Sgr A* and therefore did not include this source in their proper motion sample.
} 
use of stellar orbital motions to refine its location by a factor of 20 compared to Menten et al. (1997) and a factor of 8 compared to Reid et al. (2003). During 4 of the 9 years of this study, a star with measurable proper motions is detected within 54 mas of this location preventing a faint counterpart from being easily detected (S0-19 in 1995, S0-16 in 2000, and S0-2 in 20012002). S0-21 ( $\mathrm{K}=16.1 \mathrm{mag})$ is the only source in this study without unambiguous proper motion and its 3 measurements are all within $3 \sigma$ of the black hole's location; with only a 1

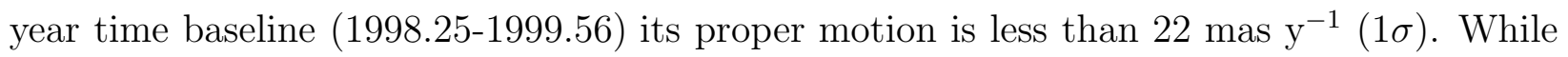
this could be a counterpart, we believe that it is not. There are 2003 correlation peaks that do not pass our 3-submap requirement, but, if real, indicate that S0-21 has measurable proper motion over this longer time baseline. In the remaining 3 years, 1996-1997 and 2003, there is no source detected by the relatively conservative source identification criteria set forth in $\S 3.1$ within $3 \sigma$ of the dynamically determined location of the black hole. We therefore infer that no steady source brighter than $\sim 15.5$, the magnitude of the faintest star we were able to identify in this region in all epochs without any a priori-information (see $\S 3.1$ ), was coincident with our inferred black hole position during our observations (see also Hornstein et al. 2002, 2003) ${ }^{4}$.

\subsection{The Origin of the Central Stellar Cluster}

The orbital parameters derived here provide important clues for understanding the origin of the Sgr A* cluster stars, which appear to have hot photospheres similar to those of massive young stars (Genzel et al. 1997; Eckart et al. 1999; Figer et al. 2000; Gezari et al. 2002; Ghez et al. 2003). In the context of the luminous He I emission line stars, which are located an order of magnitude further from the black hole than the Sgr A* cluster stars, several ideas have been proposed to account for apparently young stars in a region whose current conditions seem to be inhospitable to star formation: 1) that these are old stars masquerading as youths, 2) that they were formed more or less in-situ by a cataclysmic compression of an already dense cloud or disk, and 3) that they were formed elsewhere as part of a massive cluster, but migrated inwards rapidly by dynamical friction. Here, we briefly examine each of these hypotheses in the context of the Sgr A* cluster stars.

\footnotetext{
${ }^{4}$ We note that after submission of this paper, a variable source coincident with Sgr A* was detected at near-infrared wavelengths (Genzel et al. 2003b; Ghez et al. 2004); its characteristics are consistent with our non-detection of a steady source brighter than $15.5 \mathrm{mag}$
} 


\subsubsection{Old Stars Masquerading as Youths}

Stellar mergers of relatively old stars can, if the stellar density is sufficiently large, produce stars massive enough to appear as main-sequence OB stars. This scenario is likely to produce stars whose orbits are isotropically distributed, consistent with our observations. However, there are several challenges to this hypothesis for the stars in the Sgr A* cluster. First, several successive mergers of stars of increasing mass are required to produce a star resembling S0-2 ( O 9.5, $M \sim 15 M_{\odot}$; Ghez et al. 2003), unless the mass segregation in this region has been so strong that only stars $\gg 1 M_{\odot}$ are left. Second, as the merger products become more massive, their nuclear lifetimes decrease, so that there is less time available for the next merger event in the sequence. Using a Fokker-Planck approach, Lee (1996) investigated the stellar merger hypothesis for the massive emission-line stars in the central parsec, and concluded that an insufficient number of them is likely to be present. The Sgr A* cluster stars, however, are much more concentrated toward the center where the stellar density is maximized (Genzel et al. 2003a) and the collision time is correspondingly shorter, so in this respect, merger events may be relatively favored there. However, the third challenge is that the velocity dispersion of stars near the supermassive black hole, $400 \mathrm{~km} \mathrm{~s}^{-1}$ at 0.01 pc (e.g., Ghez et al. 1998), is comparable to the escape velocity from the surface of a main-sequence 09.5 star, $\sim 1000 \mathrm{~km} \mathrm{~s}^{-1}$, so collisions in the volume occupied by the Sgr A* cluster stars are therefore less likely to lead to mergers and mergers that do occur are likely to be accompanied by significant mass loss (Freitag \& Benz 2002). A fourth consideration which may disfavor the collisional mechanism is the relatively normal rotation rate of S0-2 (Ghez et al. 2003). Alexander \& Kumar (2001) have found that tidal encounters between main-sequence stars in the central cluster can eventually spin up those stars to near breakup speed. Colliding stars effectively represent an extreme example of this phenomenon, so merger products should be much more rapidly rotating than S0-2 appears to be. Of course, the apparent rotation rate of $\mathrm{S} 0-2$ can be attributed to a particular, low-probability orientation, so the measurement of absorption-line widths in just one additional member of the cluster should clarify this point. While further calculations are clearly required to assess the importance of this complex mechanism, at present it appears to be quite unlikely.

Another suggestion to account for the Sgr A* cluster stars without invoking star formation is that they may be exotic objects. This catch-all category includes a number of possibilities. For example, it is reasonable to expect that stellar remnants such as neutron stars and black holes sink into the central few milli-parsec as a result of dynamical mass segregation (Morris 1993; Lee 1996; Miralda-Escudé \& Gould 2000). Mergers of these remnants with normal stars could produce Thorne-Zýtkow objects, or, in the case of black hole remnants, something with dubious long-term stability. However, Thorne-Zýtkow objects are expected to appear as red giants or supergiants rather than massive blue stars, and may be 
unstable; likewise, stable stellar objects with black hole cores have yet to be described. If the stellar remnant that undergoes a merger is a white dwarf, then a "reborn star" results, and it could be suitably luminous. However, such an object would probably be a red giant rather than an early-type star, and in any case, the white dwarf precursor is likely to have migrated out of the central region because of its low mass. Another, slightly less exotic possibility for the Sgr A* cluster stars is that they be the exposed, hot cores of tidally stripped red giant stars. Indeed, there appears to be a paucity of red giants in the inner $0.2 \mathrm{pc}$ of the Galaxy (Sellgren et al. 1990; Genzel et al. 1996), suggesting that red giant atmospheres are collisionally removed there, possibly by collisions with binaries (Davies et al. 1998). However, the luminosity of the exposed stellar cores may be too small to account for the Sgr A* cluster stars (e.g., Schönberner 1981, 1983).

\subsubsection{Recent In-Situ Star Formation}

The second category of hypotheses is that the early-type stars really did form recently in situ. To do this, the parent cloud would have to have undergone violent compression to densities exceeding the limiting Roche density. ${ }^{5}$ This hypothesis warrants consideration because the mechanism for violent compression of any cloud passing close to the black hole is innate to the model. A dense cloud brought within 0.02 parsecs of the supermassive black hole would unavoidably lead to a high rate of accretion onto the black hole. If the onset of this accretion is rapid, the resultant release of accretion energy would be powerful enough to compress the cloud. Morris, Ghez \& Becklin (1999) proposed that this process can manifest itself as part of a limit cycle involving the circumnuclear disk (CND). This disk currently has a central cavity of 1 parsec radius, presumably because of the outgoing ram pressure of the winds from the cluster of luminous, early-type stars in the central parsec. However, as the lifetimes of these stars is $\sim 10^{7}$ years, and because the CND itself undergoes viscous evolution on times more comparable to the orbital time at the inner radius, $\sim 5 \times 10^{4}$ years, the inner edge of the CND will migrate toward the central black hole on a time scale comparable to the stellar evolution time. When the first portions of the CND reach the central black hole, the outgoing shock resulting from the accretion event provokes massive

\footnotetext{
${ }^{5}$ Tidal compression may be a contributor: a cloud moving toward the center on a purely radial trajectory will experience a compression in two-dimensions, although this would be partially counteracted by distension in the radial dimension, so that the net compression would not be a strong function of radius, and is not likely, by itself, to be able to raise the density by the many orders of magnitude necessary. In addition, any non-radial motion would imply a tidal shear in the azimuthal direction which would also counteract the tidal compression.
} 
star formation in the now nearby disk by strong compression. The strong winds from these stars cause the inner disk boundary to recede and the cycle begins anew. A weakness of this hypothesis is the magnitude of the required compression. While the density of a cloud which has migrated close to the black hole might be substantially larger than the densities so far inferred for any of the gas in the region, it is difficult to see how even the most effective compressive event can bring gas up to the limiting Roche density.

\subsubsection{Recent Star Formation at Large Galactic Center Distance Accompanied by Rapid Orbital Migration}

The third hypothesis which has been considered is that the early-type stars in the central parsec formed well outside the central parsec, but migrated inward under the action of dynamical friction on time scales substantially less than their nuclear time scale, $\sim 10^{7}$ years. This is not possible for individual stars (Morris 1993), but Gerhard (2001) has pointed out that, because the dynamical friction time scale is inversely proportional to an object's mass, sufficiently massive clusters can migrate to the central parsec from radii of tens of parsecs within the required time, especially if they remain bound to their parent cloud. This hypothesis has been investigated numerically by Kim \& Morris (2003) and Portegies-Zwart, McMillian \& Gerhard (2003), who clarify that very massive clusters are required $-10^{5-6} \mathrm{M}_{\odot}$, far more massive than even the extreme (for our Galaxy) Arches and Quintuplet clusters (e.g., Figer, McLean, \& Morris 1999; Figer et al. 2002). Core collapse is inevitable in the massive, dense clusters required for the cluster inspiral hypothesis. This process helps ensure that, in spite of tidal stripping of stars outside the cluster core as the cluster migrates inward, there remains a tightly bound, cluster core that survives intact into the central parsec. However, Kim \& Morris find that the mass of stars reaching the central parsec, for any feasible initial cluster mass, substantially exceeds the mass of early-type stars in the central parsec cluster.

More recently, Portegies-Zwart and McMillan (2002, see also Rasio, Freitag, \& Gürkan 2004) have raised the possibility that core collapse in sufficiently massive clusters proceeds all the way to the formation of an intermediate-mass black hole (IBH), which can carry cluster stars in with it as it spirals inward by dynamical friction. The implications of such a cluster-produced IBH for the distribution of early-type stars in the central parsec have recently been investigated by Hansen \& Milosavljević (2003). They argue that the HeI emission-line stars in the central parsec have been tidally stripped from the IBH during successive passages near the supermassive black hole, but that they retain a memory of the IBH orbit. Furthermore, cluster evaporation during the inspiralling process leads to a marked decrease in the effectiveness of this process; a remnant core of an initially globular-cluster- 
mass cluster can reach the central parsec only by distributing a large number of early-type stars at all radii, whereas there is currently no evidence for a young population beyond the central parsec. In addition, Kim, Figer, \& Morris (2004) find that an IBH helps deliver stars to the central parsec only if it contains at least $10 \%$ of the cluster mass, far larger than masses obtained in simulations of successive merger (Portegies-Zwart \& McMillan). Further investigations of this hypothesis are warranted, though it currently appears to suffer from a number of difficulties.

Both the in situ formation mechanism and the evaporating, inspiralling cluster mechanism will lead primarily to a disk of stars, the first because the inwardly migrating reservoir of gas inevitably forms a disk by virtue of its angular momentum, and the second because the stars lost from the cluster will retain a memory of the direction of the cluster angular momentum. While most of the early-type emission-line stars in the central parsec appear to orbit in or near a well-defined plane (Levin \& Beloborodov 2003), the stars in the SgrA* cluster do not. Levin \& Beloborodov argue that the SgrA* cluster stars and the more distant emission-line stars all formed at about the same time in a starburst taking place in a thick accretion disk around $\operatorname{SgrA}^{*}$ (see also Nayakshin \& Cuadra 2004). Unlike the He-I emission line stars, the orbits of the SgrA* cluster stars are likely to have been altered by Lens-Thirring precession caused by the massive central black hole, so that their orbital angular momenta vectors should form a plane, which is inconsistent with the observations (see Figure 3). This suggests that the Sgr A* cluster stars were not formed by these mechanisms, which produce an initial common direction for the angular momentum vector.

One alternative hypothesis for the tight orbits of the SgrA* cluster stars is that they have resulted from the tidal disruption of massive star binaries as stars presumably related to the HeI emission-line stars undergo relatively close passages by the supermassive black hole (Gould \& Quillen 2003). These authors estimate that a sufficient number of single stars resembling S0-2 can be scattered onto orbits similar to those of the SgrA* cluster stars to explain that cluster, if they originate in binary systems undergoing close passage by the black hole. Multiple encounters with other stars in this region are required to bring the apoapse distances down to the range of values exhibited by the SgrA* cluster stars, i.e., far smaller than the typical orbital radii of the more massive emission-line stars. It remains to be seen whether this hypothesis can account for the SgrA* cluster stars.

In sum, there are serious difficulties or open questions associated with all of these hypotheses, although few of them can be definitively ruled out. While the stars with known orbits offer modest support for hypotheses that produce isotropic distributions, this is based on a very small sample. Additional orbits for stars in the vicinity of the central black hole may ultimately provide a sufficiently strong constraint to cull this list of possibilities. In the 
meantime, we are left with an interesting conundrum.

\section{Conclusions}

After almost a decade of diffraction-limited imaging at the W. M. Keck I 10-meter telescope, we have obtained orbital solutions for multiple stars. This orbital analysis has the advantage of simultaneously solving for a common set of properties for the central dark object, which not only reduces the uncertainties in the black hole's mass and location compared to an analysis that treats each star independently, but also provides the first direct measure of the black hole's velocity with respect to the central stellar cluster. Together, the stellar motions reveal a central dark mass of $3.7( \pm 0.2) \times 10^{6}\left(\frac{R_{o}}{8 k p c}\right)^{3} M_{\odot}$ and confine it to within a radius of a mere $45 \mathrm{AU}$ or equivalently $600 \mathrm{R}_{s h}$, dramatically strengthening the case for a supermassive black hole, the location of which is now determined to within \pm 1.3 mas (10 $\mathrm{AU})$. Consequently, the dark mass at the center of the Milky Way has become the most ironclad case of a supermassive black hole at the center of any normal type galaxy.

The precision of the proper motion and radial velocity measurements opens up additional new realms for dynamical studies in the Galactic Center. First is the possibility of doing a full orbital model, which also solves for the distance to the central black hole as well as its motion along the line of sight (Salim \& Gould 1999; Ghez et al. 2003). While solving for the motion along the line of sight will require several more years of radial velocity data on preferably several stars, only one more year of both astrometric measurements and radial velocity measurements for S0-2 alone should provide the most direct and precise estimate of the distance to the Galactic Center (see, e.g., Eisenhauer et al. 2003). A second opportunity is the possibility of detecting deviations from a Keplerian orbit. These might arise from precession of the periapse distance due to general relativistic effects (Jaroszynski 1998; Fragile \& Matthews 2000), which would require the discovery of a star with a significantly smaller periapse passage than has been found so far, or, more likely, an extended mass distribution (Rubilar \& Eckart 2001), in the form of either an entourage of stellar remnants surrounding the central supermassive black hole, a spike of dark matter particles (Gondolo \& Silk 1999; Ullio et al. 2001; Gnedin \& Primack 2004) or a binary black hole.

The stars that have been the tracers of the gravitational potential are themselves quite interesting. Their spectral features suggest that they are young $(<10 \mathrm{Myr})$. Since these stars currently reside in a region that is inhospitable to star formation, they are either old stars whose appearance has been significantly altered or they are young stars formed by a mechanism that is able to circumvent the challenges presented by the central black hole. This study, for the first time, uses the kinematics of stars in the Sgr A* cluster to shed 
light on this paradox. Among the notable properties are eccentricity, angular momentum, and apoapse distributions that show no statistically significant departures from an isotropic distribution. This differs significantly from the He-I emission line stars, which appear to be co-planar. It therefore appears that the two populations of young stars in the vicinity of the Galactic Center black hole - the Sgr A* cluster stars and the He-I emission line stars formed by different mechanisms. In particular, it is unlikely that the Sgr A* cluster stars formed from a disk. Additional orbits will help to clarify the ensemble kinematics of this unusual group of stars, which reside in a particular complex region.

The authors thank Joel Aycock, Randy Campbell, Bob Goodrich, David LeMignant, Chuck Sorensen, and Peter Wizinowich at the Keck Observatory for their help in obtaining the new observations, Gary Chanan for making it possible to phase the Keck Telescope on NIRC, Mark Reid for advance information on IRS 9 and 12N, Mike Jura, Brad Hansen, Raoul Viollier, and Shelley Wright for helpful conversations, and an anonymous referee for a helpful review. Support for this work was provided by the National Science Foundation grant AST9988397 and the National Science Foundation Science and Technology Center for Adaptive Optics, managed by the University of California at Santa Cruz under cooperative agreement No. AST-9876783, and the Packard Foundation. The W.M. Keck Observatory is operated as a scientific partnership among the California Institute of Technology, the University of California and the National Aeronautics and Space Administration. The Observatory was made possible by the generous financial support of the W.M. Keck Foundation. The authors also wish to recognize and acknowledge the very significant cultural role and reverence that the summit of Mauna Kea has always had within the indigenous Hawaiian community. We are most fortunate to have the opportunity to conduct observations from this mountain.

\section{A. Source Naming}

Newly identified sources are named here using the convention introduced by Ghez et al. (1998), which was designed to directly convey relevant information about the location of the source relative to the position of Sgr A*. Originally, the Sgr A* position given by Menten et al. (1997) was adopted and the surrounding field was divided into concentric arcsecond-wide annuli centered on this position. Stars lying within the central circle, which has a radius of 1 arcsecond, were given names S0-1, S0-2, S0-3, etc. Stars lying in the annulus between radii of 1 to 2 arcseconds were given the names S1-1, S1-2, and so on. The number immediately following " $S$ " thus refers to the inner radius of the annulus in which the star lies. The number following the hyphen was ordered in the sense of increasing distance from Sgr A* within each annulus at the time of its naming. In this scheme, newly identified sources are named by 
incrementing the number following the hyphen within each annulus and ordered in the sense of increasing distance from Sgr $\mathrm{A}^{*}$ at the time of discovery. Since the original list within 1 arcsecond ended at 15, the newly identified stars begin with 16 . S0-16, S0-17, and S0-18 were labeled by us in a recent spectroscopic paper (Gezari et al. 2001) and S0-19 and S0-20 were first presented at the Rees Symposium "Making Light of Gravity," held in Cambridge, England (July 2002). Due to the motions of stars, and refinements in the location of Sgr $\mathrm{A}^{*}$, the current distance rank does not necessarily match the one at the time of discovery.

\section{B. Absolute Astrometry}

Estimates of the camera's pixel scale and orientation, as well the position of Sgr A*, require tying the relative measurements to an absolute reference frame. This was done by obtaining multiple telescope pointings that allow the construction of mosaics covering the positions of Sgr A* and two SiO masers, IRS 7 and IRS 10EE in 1998 May, 1998 Aug, 1999 May, 1999 July, 2000 May, 2000 July, 2001 May, 2001 July. In 1999 July, a somewhat larger region was covered to include the positions of two additional masers, IRS 9 and 12N. By combining our infrared astrometry with radio astrometry from Reid et al. (2003), we derive a pixel scale, $20.396 \pm 0.042$ mas $\mathrm{pix}^{-1}$, a position angle of North with respect to NIRC columns in 1999 July, $0.80 \pm 0.14$ degrees, and a location of Sgr A*, which is located to within \pm 6.4 (E-W) mas and 14 (N-S) mas; Table 4 lists the positions, with respect to Sgr $\mathrm{A}^{*}$, of IRS $16 \mathrm{NW}$ and IRS $16 \mathrm{C}$.

The infrared positional uncertainties obtained in this procedure are larger than can be explained by uncertainties in the infrared centroids of these bright stars or the alignment of the map to a common epoch. This, most likely, reflects a small residual distortion in the NIRC camera ${ }^{6}$. The effects of distortion are minimized in our measurements of the Sgr $\mathrm{A}^{*}$ cluster stars by always positioning them at the center of the field of view and carrying out the observations over similar ranges of parallactic angle during every observing run ${ }^{7}$. In contrast, the masers not only occupied different camera positions, but were measured at different times during the night, resulting in non-constant relative position vectors on the camera between each maser and Sgr A* from run to run. This, unlike the measurements

\footnotetext{
${ }^{6} \mathrm{~A}$ known distortion in the NIRC optics is corrected for in the individual exposures before the SAA maps are made, however any distortion introduced by the reimager (Matthews et al. 1996) has not been accounted for and is the likely source of additional measurement error.

${ }^{7}$ The rotator was turned off during this experiment so the direction of North with respect to the camera, the parallactic angle, changes throughout the night.
} 
of the stars within the Sgr A* cluster, maximally sampled the effects of distortion, which amount to a $\sim 0.3$ pixel offset from the center of the field of view to the edge (a $0.2 \%$ effect). This distortion is what dominates our uncertainties in the inferred infrared position of Sgr $A^{*}$ ( \pm 6 mas), which are nonetheless almost a factor of two smaller that that obtained by Reid et al. (2003, \pm 10 mas) in their infrared reference frame. In contrast, the distortion is not a significant effect for the relative stellar position measurements of stars in Table 1, which have a maximum displacement of $\sim 0^{\prime \prime} .3$ over the course of this study and therefore experience at most a $\sim 0.6$ mas offset from distortion.

\section{REFERENCES}

Alexander, T. \& Kumar, P. 2001, ApJ, 549, 948

Backer, D. C., \& Sramek, R. A. 1999, ApJ, 524, 805

Binney, J. \& Tremaine, S. 1987, Princeton, NJ, Princeton University Press, 1987, 747

Chakrabarty, D., \& Sarah, P. 2001, AJ, 122, 232

Christopher, M. H. \& Scoville, N. Z. 2003, ASP Conf. Ser. 290: Active Galactic Nuclei: From Central Engine to Host Galaxy, 389

Davies, M. B., Blackwell, R., Bailey, V. C., Sigurdsson, S. 1998, MNRAS, 301, 745

Diolaiti, E., Bendinelli, O., Bonaccini, D., Close, L., Currie, D., \& Parmeggiani, G. 2000, A\&AS, 147, 335

Eckart, A., \& Genzel, R. 1997, MNRAS, 284, 576

Eckart, A., Genzel, R., Ott, T., \& Schödel, R. 2002, MNRAS, 331, 917

Eckart, A., Ott, T., \& Genzel, R. 1999, A\&A, 352, L22

Eisenhauer, F., Schödel, R., Genzel, R., Ott, T., Tecza, M., Abuter, R., Eckart, A., \& Alexander, T. 2003, ApJ, 597, L121

Ferrarese, L., \& Merritt, D. 2000, ApJ, 539, L9

Fragile, P. C., \& Mathews, J. 2000, ApJ, 542, 328

Freitag, M., \& Benz, W. 2002, A\&A, 394, 345

Figer, D. F. et al. 2000, ApJ, 533, L49 
Figer, D. F., McLean, I. S., \& Morris, M. 1999, ApJ, 514, 202

Figer, D. F., et al. 2002, ApJ, 581, 258

Figer, D. F., et al. 2003, ApJ, 599, 1139

Figer, D. 1995, Ph.D. Thesis, University of California, Los Angeles

Gebhardt, K. et al. 2000, ApJ, 539, L13.

Genzel, R., Eckart, A., Ott, T., \& Eisenhauer, F. 1997, MNRAS, 2 91, 219

Genzel, R., Pichon, C., Eckart, A., Gerhard, O. E., Ott, T., 2000, MNRAS, 317, 348

Genzel, R. et al. 2003a, ApJ, 594, 812

Genzel, R. Schödel, R., Ott, T., Eckart, A., Alexander, T., Lacombe, F., Rouan, D., \& Aschenbach, B. 2003b, Nature, 425, 934

Genzel, R., Thatte, M., Krabbe, Kroker, H., \& Tacconi-Garman, L. E. 1996, ApJ, 472, 153

Gerhard, O. 2001, ApJ, 546, L39

Gezari, S., Ghez, A. M., Becklin, E. E., Larkin, J., McLean, I. S., Morris, M. 2002, ApJ, 576,790

Ghez, A. M., Duchêne, G., Matthews, K., Hornstein, S. D., Tanner, A., Larkin, J., Morris, M., Becklin, E. E., Salim, S., Kremenek, T., Thompson, D., Soifer, B.T., Neugebauer, G., McLean, I. 2003, ApJ, 586, L127

Ghez, A. M., Klein, B. C., Morris, M., \& Becklin, E. E. 1998, ApJ, 509, 678

Ghez, A. M., Morris, M., Becklin, E. E., Tanner, A., \& Kremenek, T. 2000, Nature, 407, 349

Ghez, A.M., Wright, S.A., Matthews, K., Thompson, D., LeMignant, D., Tanner, A., Hornstein, S.D., Morris, M., Becklin, \& Soifer, B. T. 2004 ApJ, 601, L159

Gondolo, P., \& Silk, J. 1999, Phys. Rev. Lett., 83, 1719

Gould, A., \& Quillen, A. C. 2003, ApJ, 592, 935

Gnedin, O. Y. \& Primack, J. R. 2004, Physical Review Letters, 93, 061302

Greenhill, L. J., Jiang, D. R., Moran, J. M., Reid, M. J., Lo, K. Y., Claussen, M. J. 1995, ApJ, 440, 619 
Haller, J. W., Rieke, M. J., Rieke, G. H., Tamblyn, P., Close, L., \& Melia, F. 1996, ApJ, 456, 194

Hansen, B. M. S., \& Milosavljević, M. 2003, ApJ, 593, L77

Hornstein, S. D., Ghez, A. M., Tanner, A., Morris, M., Becklin, E. E., Wizinowich, P, 2002, ApJ, 577, L9

Hornstein, S. D., Ghez, A. M., Tanner, A., Morris, M., \& Becklin, E. E. 2003, "Limits on the Short Term Variability of Sagittarius A* in the Near Infrared," Astron. Nachr., Vol. 324, No. S1, Special Supplement "The central 300 parsecs of the Milky Way", Eds. A. Cotera, H. Falcke, T. R. Geballe, S. Markoff

Jackson, J. M., Geis, N., Genzel, R., Harris, A. I., Madden, S., Poglitsch, A., Stacey, G. J., Townes, C. H. 1993, ApJ, 402, 173

Jaroszynski, M. 1998, Acta Astronomica, 48, 653.

Kim, S. S., Figer, D. F., \& Morris, M. 2004, ApJ, 607, L123.

Kim, S. S., \& Morris, M. 2003, ApJ, 597, 312

Lacy, J. H., Townes, C. H., Geballe, T. R., \& Hollenbach, D. J., 1980, ApJ, 241, 132

Lee, H.M., 1996, IAU 169, 215

Levin, Y., \& Beloborodov 2003, ApJ, 590, L33

Lo, K. Y., Backer, D. C., Ekers, R. D., Kellermann, K. I., Reid, M., \& Moran, J. M. 1985, Nature, 315, 124

Maoz, E. 1998, ApJ, 494, L181

Matthews, K., Ghez, A. M., Weinberger, A. J., and Neugebauer, G. 1996, PASP, 108, 615

Matthews, K. and Soifer, B. T. 1994, Astronomy with Infrared Arrays: The Next Generation, ed. I. McLean, Kluwer Academic Publications (Astrophysics and Space Science, v. 190, p. 239)

McGinn, M. T., Sellgren, K., Becklin, E. E., \& Hall, D. N. B., 1989, ApJ, 338, 82

Menten, K. M., Reid, M. J., Eckart, A., \& Genzel, R. 1997, ApJ, 475, L111

Merritt, D., Ferrarese, L. 2001, ApJ, 547, 140 
Miralda-Escudé, J., \& Gould, A. 2000, ApJ, 545, 847

Morris, M., 1993, ApJ, 408, 496

Morris, M., Ghez, A. M., Becklin, E. E. 1999, Adv. Spa. Res., 23, 959

Miyoshi, M., Moran, J. M., Hernstein, J., Greenhill, L., Nakai, N., Diamond, P., \& Inoue, M. 1995, Nature, 373, 127

Munyaneza, F. \& Viollier, R. D. 2002, ApJ, 564, 274.

Nayaksin, S. \& Cuadra, J. 2004, A\&A, submitted (astro-ph/0409541)

Portegies Zwart, S. F., \& McMillian, S. L. W. 2002, ApJ, 576, 899

Portegies Zwart, S. F., McMillian, S. L. W., Gerhard, O. 2003, ApJ, 593, 352.

Rasio, F. A., Freitag, M., \& Gürkan, M. A. 2004, Coevolution of Black Holes and Galaxies, 138

Reid, M. J. 1993, ARA\&A, 31, 345

Ried, M. J. \& Brunthaler, A. 2004, ApJ, accepted (astro-ph/0408107)

Reid, M. J., Menten, K. M., Genzel, R., Ott, T., Schödel, R., \& Eckart, A. 2003, ApJ, 587, 208

Reid, M. J., Readhead, A. C. S., Vermeulen, R. C., Treuhaft, R. N. 1999, ApJ, 524, 816

Rubilar, G. F., \& Eckart, A. 2001, A\&A, 2001, 372, 95

Salim, S., \& Gould, A. 1999, ApJ, 523, 633

Sanders, R. H. 1992, Nature, 359, 131

Schönberner, D. 1981, A\&A, 103, 119

Schönberner, D. 1983, ApJ, 272, 708

Schödel, R. et al. 2002, Nature, 419, 694

Schödel, R., Ott, T., Genzel, R., Eckart, A., Mouawad, N., \& Alexander, T. 2003, ApJ, 596, 1015

Scoville, N. Z., Stolovy, S. R., Rieke, M., Christopher, M. H., Yusef-Zadeh, F. 2003, ApJ, 594,294 
Sellgren, K., McGinn, M. T., Becklin, E. E., \& Hall, D. N. B. 1990, ApJ, 359, 112

Spergel, D. N. et al. 2003, ApJ, submitted

Tanner, A. 2004, PhD UCLA

Tremaine, S.. et al. 2002, ApJ, 574, 740

Tsiklauri, D., \& Viollier, R. D. 1998, ApJ, 500, 591

Ullio, P., Zhao, H., Kamionkowski, M. 2001, Phys. Rev. D, 64, 1302

Viollier, R. 2003, Astron. Nachr., Vol. 324, No. S1, Special Supplement "The central 300 parsecs of the Milky Way", Eds. A. Cotera, H. Falcke, T. R. Geballe, S. Markoff 


\section{Galactic Center 2000 May / Keck}

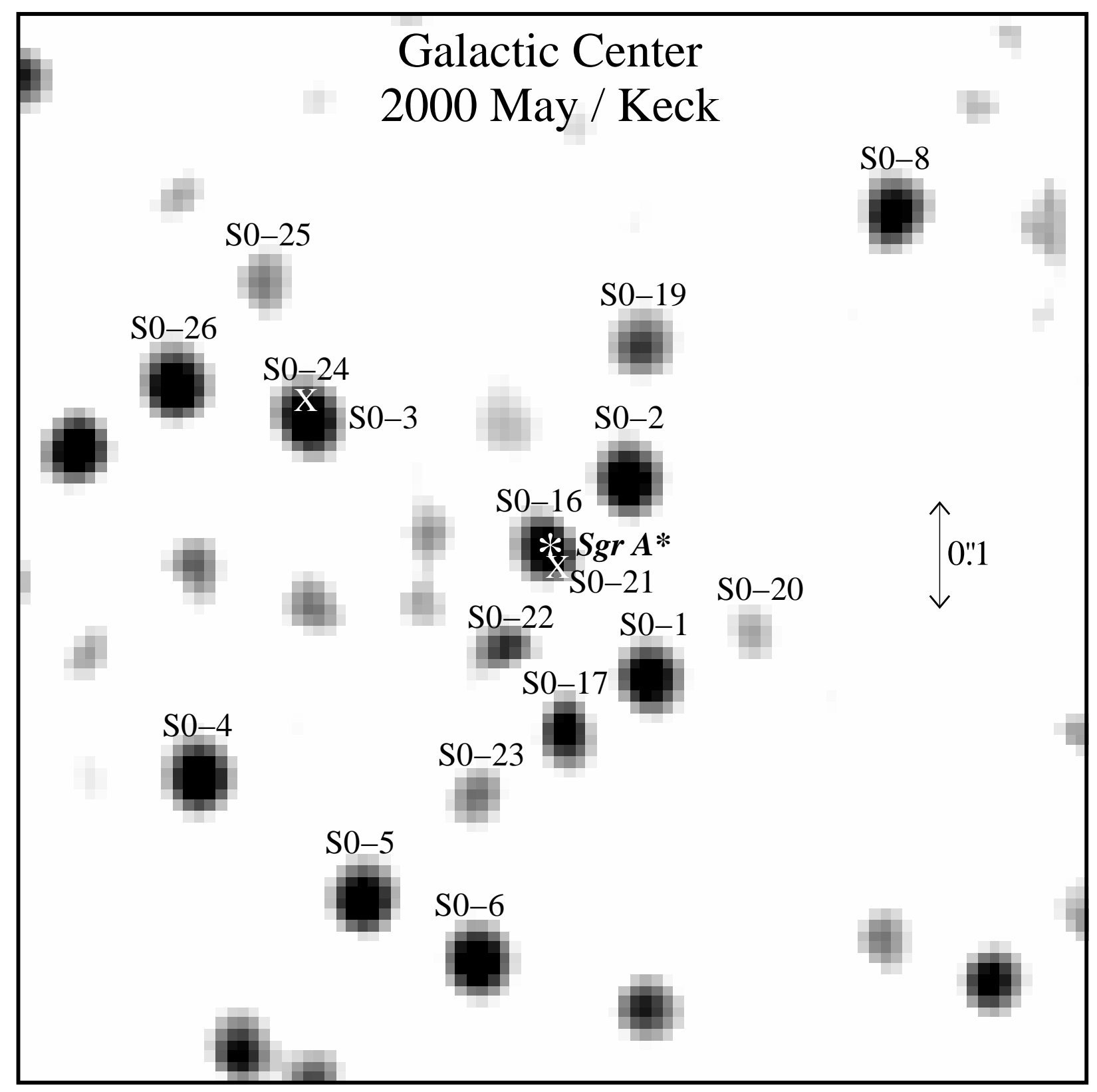

Fig. 1.- The central $1^{\prime \prime} \times 1^{\prime \prime}$ of the cross-correlation (or match filter) map for the 2000 May data set. Of the 17 sources identified in this study by the criteria described in $\S 3.1,15$ are seen in this map. The remaining 2, marked with crosses, are missed in this particular map due to confusion with a brighter nearby source. An asterisk denotes the black hole's dynamically determined position (see §3). The criteria used for source identification are still quite conservative as there are several unlabeled peaks that appear to be real sources, within 0'.4 of Sgr A*. 


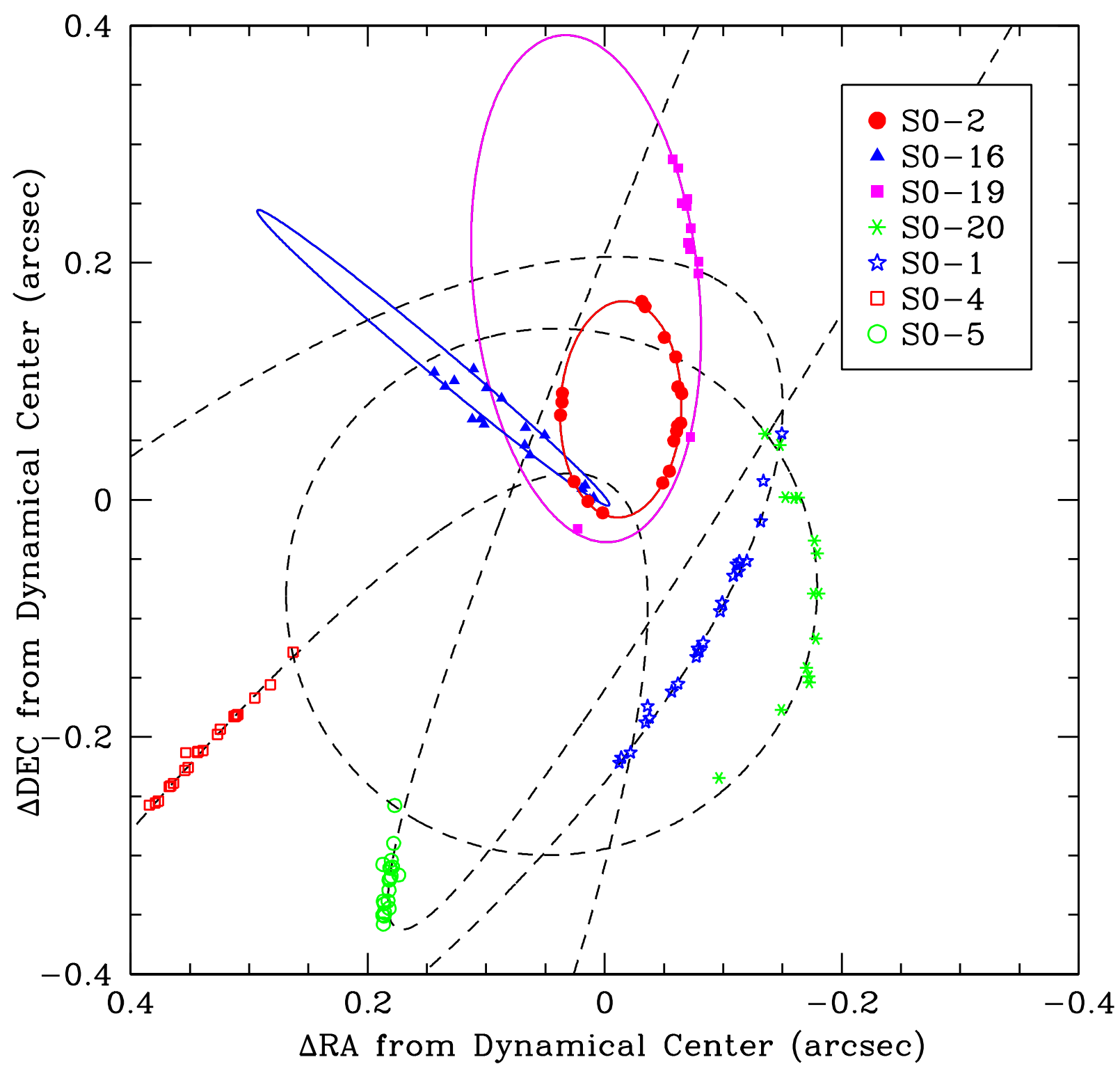

Fig. 2.- Astrometric positions and orbital fits for the 7 stars that show significant deviation from linear motion. The proper motion measurements were obtained between 1995 and 2003 at the Keck telescopes, have uncertainties that are comparable to or smaller than the size of the points, and are plotted in the reference frame in which the central dark mass' is at rest. On the plane of the sky, three of these stars show orbital motion in the clockwise direction (S0-1, S0-2, and S0-16) and four of these stars have counterclockwise motion (S0-4, S0-5, S0-19, and S0-20). Overlaid are the best fitting simultaneous orbital solutions, which assume that all the stars are orbiting the same central point mass. The orbital solutions for the three stars that constrain the properties of the central dark object are delineated by solid lines and the joint orbital solutions for the remaining stars are shown with dashed lines. 


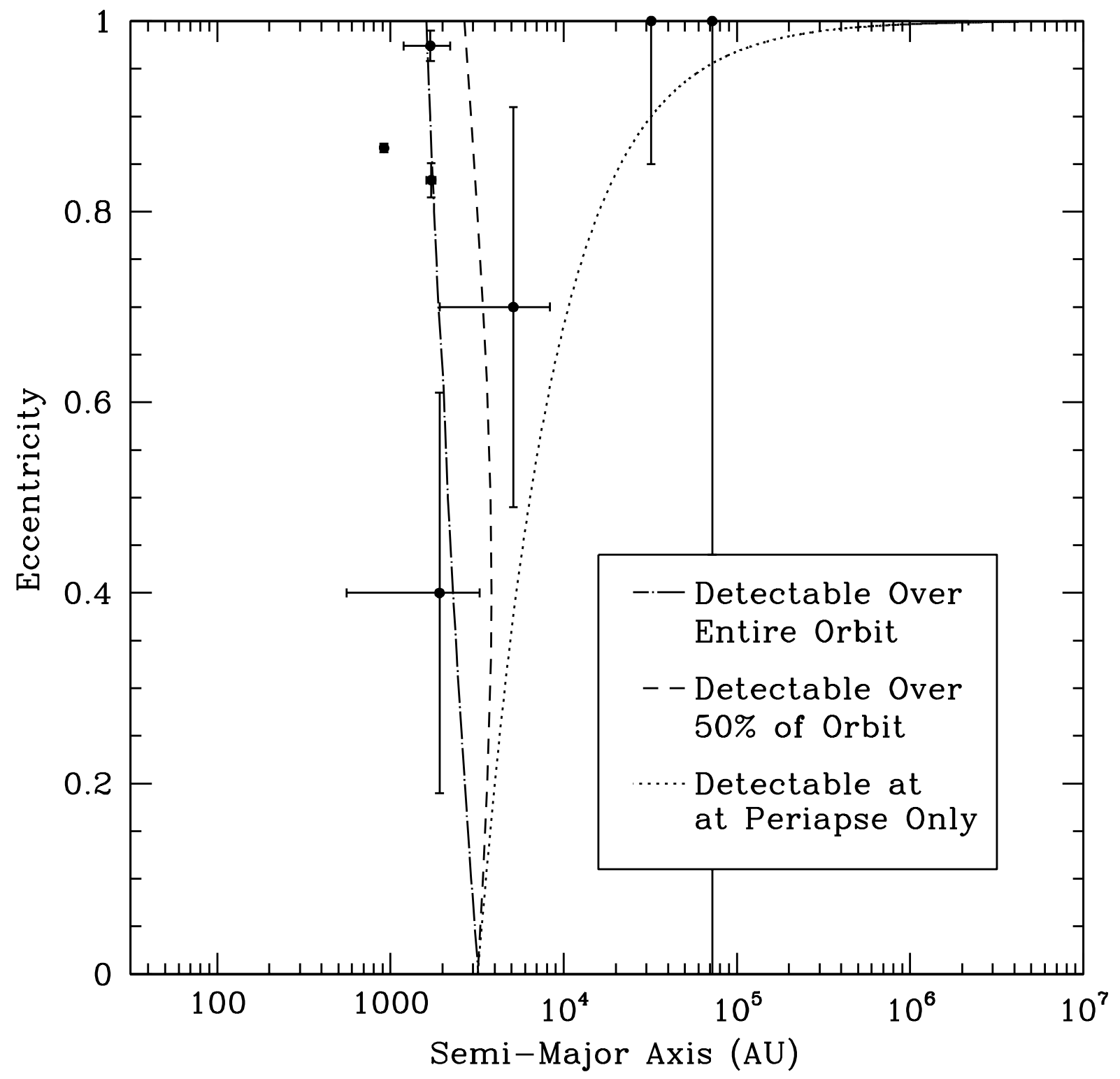

Fig. 3.- Eccentricity vs. Semi-Major Axis for the 7 stars included in this study. The observational selection effects are quantified by the fraction of time a face-on orbit experiences acceleration larger than our threshold value of 2 mas $\mathrm{y}^{-2}$, with the cases of $100 \%$ of the orbit (long dashed - dotted line), 50\% of the orbit (short dashed line), and only periapse passage (dotted line) shown. The region to the left of the long dashed - dotted line is free of observational selection effects and therefore should not be missing any stars that are brighter than $\sim 15.5$. 


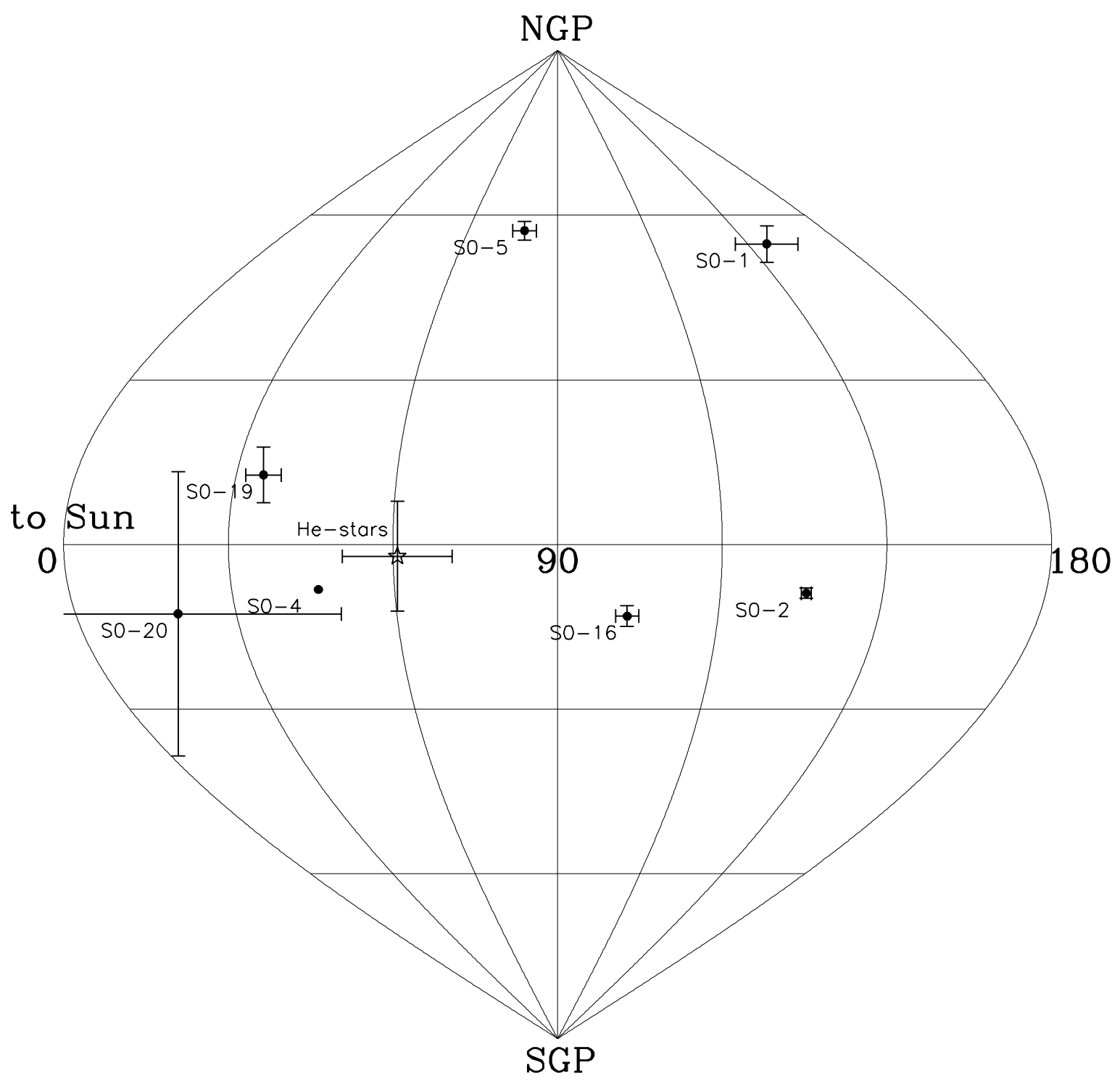

Fig. 4.- Positions of the angular momentum vectors for an observer at the center of the Galaxy. Only one hemisphere is shown (East as seen from the Earth), since the degeneracy of the inclination sign makes it impossible to know which hemisphere a vector points, except for S0-2. The large uncertainties for S0-4 have been omitted for clarity. If the orbits were to be co-planar the angular momentum vectors would cluster, which we do not see. The normal to the plane of the He I stars found by Levin \& Beloborodov (2003) is also indicated. 


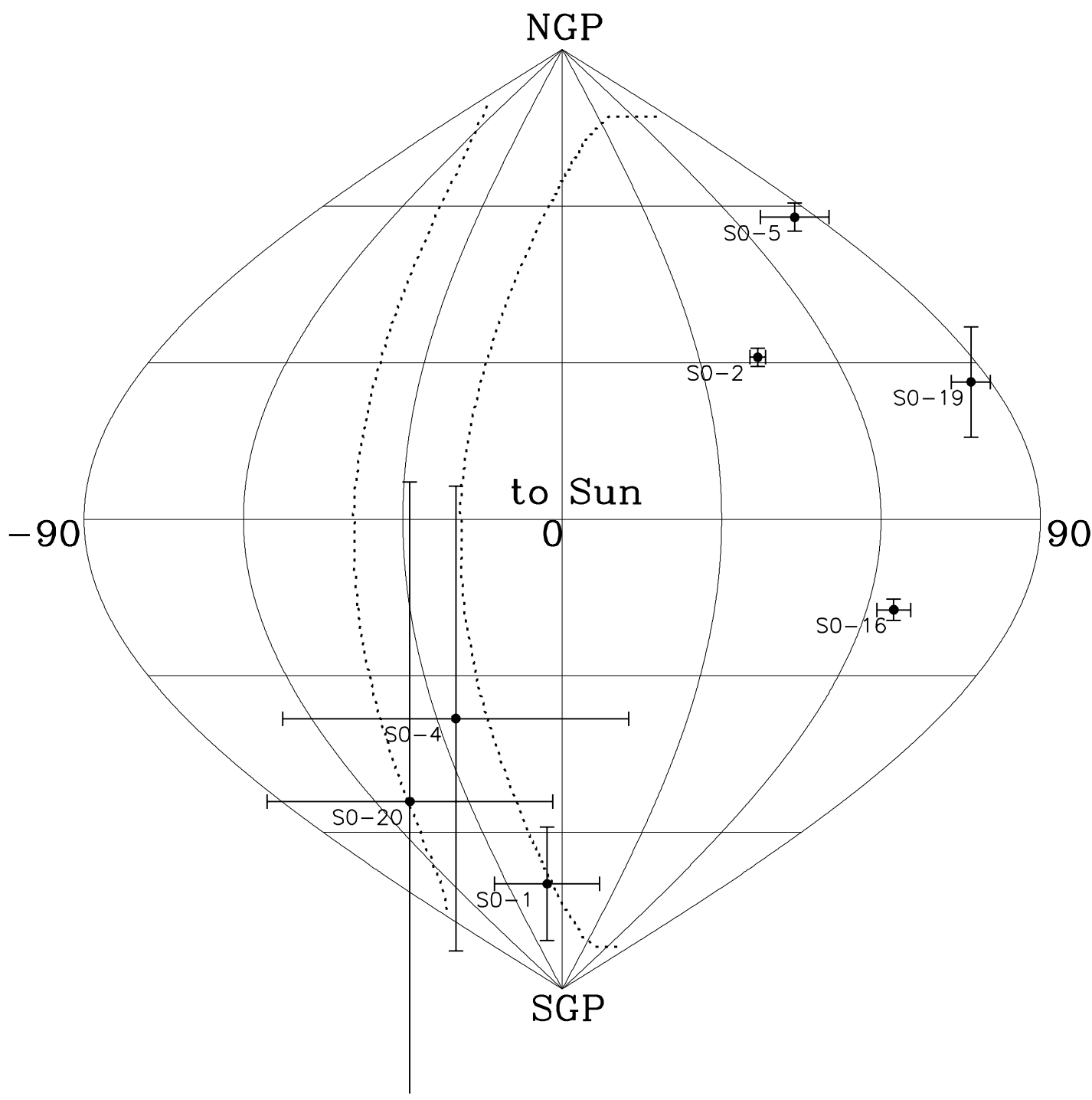

Fig. 5.- The directions of the apoapse vectors, as seen from an observer at the Galactic center, for the hemisphere containing S0-2. The He I star plane of Levin \& Beloborodov (2003) is shown as a $20^{\circ}$ wide band (dotted lines). These vectors are consistent with an isotropic distribution, even when the degeneracy of the inclination sign is taken into account. 
Table 1. Summary of Sources Identified within 0.'4 of the Central Dark Mass

\begin{tabular}{|c|c|c|c|c|c|c|c|c|c|c|c|}
\hline \multirow{3}{*}{$\begin{array}{c}\text { Star } \\
\text { Name }\end{array}$} & \multirow{3}{*}{$\begin{array}{l}\text { Other } \\
\text { Name }\end{array}$} & \multirow{3}{*}{$\begin{array}{l}<K> \\
(\mathrm{mag})\end{array}$} & \multicolumn{2}{|c|}{ \# Epochs } & \multicolumn{4}{|c|}{ Closest Measured Position ${ }^{b}$} & \multirow{3}{*}{$\begin{array}{c}<\sigma_{\text {pos }}> \\
(\operatorname{arcsec})\end{array}$} & \multirow[t]{3}{*}{$\Delta \chi^{2 \mathrm{c}}$} & \multirow[t]{3}{*}{ Notes } \\
\hline & & & $N_{\text {det }}$ & $N_{f i t}$ & Date & $\mathrm{R}$ & $\Delta \mathrm{RA}$ & $\Delta \mathrm{Dec}$ & & & \\
\hline & & & & & (year) & $(\operatorname{arcsec})$ & $(\operatorname{arcsec})$ & $(\operatorname{arcsec})$ & & & \\
\hline S0-2 & $\mathrm{S} 2$ & $14.0 \pm 0.2$ & 22 & $18^{\mathrm{f}}$ & 2000.309 & 0.012 & -0.065 & 0.059 & 0.001 & 6132 & \\
\hline S0-16 & $\mathrm{S} 14$ & $15.5 \pm 0.3$ & $18^{\mathrm{d}}$ & $17^{\mathrm{f}}$ & 2000.305 & 0.006 & 0.005 & -0.001 & 0.002 & 3570 & $\mathrm{New}^{\mathrm{g}}$ \\
\hline S0-19 & $\mathrm{S} 12$ & $15.5 \pm 0.2$ & $13^{\mathrm{d}, e}$ & $12^{\mathrm{f}}$ & 1995.439 & 0.036 & 0.015 & -0.033 & 0.002 & 936 & $\mathrm{New}^{\mathrm{g}}$ \\
\hline S0-20 & S13 & $15.7 \pm 0.2$ & $15^{\mathrm{e}}$ & 15 & 2003.682 & 0.147 & -0.136 & 0.057 & 0.004 & 286 & $\mathrm{New}^{\mathrm{g}}$ \\
\hline S0-1 & $\mathrm{S} 1$ & $14.6 \pm 0.1$ & 22 & 22 & 1998.505 & 0.131 & -0.117 & -0.060 & 0.001 & 281 & \\
\hline $\mathrm{S} 0-4$ & $\mathrm{~S} 8$ & $14.4 \pm 0.1$ & 22 & $21^{\mathrm{f}}$ & 1995.439 & 0.290 & 0.255 & -0.137 & 0.001 & 53 & \\
\hline S0-5 & S9 & $15.1 \pm 0.2$ & $21^{\mathrm{e}}$ & $20^{\mathrm{f}}$ & 1995.439 & 0.316 & 0.169 & -0.267 & 0.002 & 35 & \\
\hline S0-23 & ID7 & $16.7 \pm 0.2$ & $9^{\mathrm{d}, e}$ & 9 & 1996.485 & 0.157 & -0.024 & -0.155 & 0.005 & 14 & $\mathrm{New}^{\mathrm{g}}$ \\
\hline S0-25 & ID9 & $16.4 \pm 0.3$ & $11^{\mathrm{e}}$ & 11 & 1998.771 & 0.364 & 0.262 & 0.253 & 0.006 & 10 & $\mathrm{New}^{\mathrm{g}}$ \\
\hline S0-8 & ID14 & $15.7 \pm 0.2$ & $20^{\mathrm{e}}$ & 20 & 2003.303 & 0.390 & -0.296 & 0.253 & 0.003 & 7 & New \\
\hline S0-17 & $\ldots$ & $15.8 \pm 0.2$ & $16^{\mathrm{e}}$ & $6^{\mathrm{f}}$ & 2003.682 & 0.115 & 0.028 & -0.112 & 0.004 & 6 & New \\
\hline S0-26 & ID12 & $15.1 \pm 0.2$ & $19^{\mathrm{d}, e}$ & 19 & 1997.367 & 0.385 & 0.366 & 0.120 & 0.002 & 6 & $\mathrm{New}^{\mathrm{g}}$ \\
\hline S0-22 & $\ldots$ & $16.8 \pm 0.4$ & $7^{\mathrm{d}, e}$ & 7 & 2001.572 & 0.093 & 0.031 & -0.088 & 0.01 & 5 & New \\
\hline S0-24 & $\ldots$ & $15.7 \pm 0.2$ & $5^{\mathrm{d}}$ & 5 & 1998.505 & 0.283 & 0.244 & 0.142 & 0.008 & 2 & New \\
\hline S0-6 & $\mathrm{S} 10$ & $14.2 \pm 0.1$ & 22 & 22 & 2003.682 & 0.378 & 0.058 & -0.374 & 0.001 & 0 & \\
\hline S0-21 & $\ldots$ & $16.1 \pm 0.3$ & $3^{\mathrm{h}}$ & $\ldots$ & 1999.560 & 0.009 & -0.007 & -0.006 & 0.006 & $\ldots$ & New \\
\hline S0-3 & $\mathrm{S} 4$ & $14.4 \pm 0.2$ & 22 & $\ldots \mathrm{f}$ & 1995.439 & 0.180 & 0.149 & 0.101 & 0.001 & $\ldots$ & \\
\hline
\end{tabular}

${ }^{\text {a } O t h e r ~ n a m e s ~ t a k e n ~ f r o m ~ E c k a r t ~ \& ~ G e n z e l ~(1997) ~ a n d ~ S c h o ̈ d e l ~ e t ~ a l . ~(2003) . ~}$

${ }^{\mathrm{b}}$ Minimum measured projected separation (1995 - 2003) from the dynamical center, whose location is reported in $\S 3.3$ and Table 2 .

${ }^{\mathrm{c}} \Delta \chi^{2}$ is the difference between the total $\chi^{2}$ value resulting from the best linear fit and the total $\chi^{2}$ from the best second order polynomial fit. Sources with $\Delta \chi^{2}$ greater than 15 are considered to have significant proper motions accelerations.

dThe following stars have missing measurements due to stellar confusion: S0-16 (1995 June - 1997 May) due to S0-3, S0-19 (1998 April - 1999 May) due to S0-2, S0-22 (2002 July - 2003 September) due to S0-17, S0-23 (1997 May - 1998 October ) due to S0-17, S0-24 (1998 May, 1998 August - 2003 September) due to S0-3, and S0-26 (1995 June - 19996 June) due to S0-7.

eThe following stars have missing measurements that are likely due to insufficient map sensitivity (either in the main map or in at least one of the sub-maps; see §2): S0-5 (1996 June), S0-19 (1996 June, 2000 April, 2003 April), S0-20 (1996 June, 1998 April, May, 1999 May, 2000 July, October 2003 July), S0-8 (1996 June \& 1998 April), S0-17 (1995 June, 1997 May, 2000 October, 2001 July, 2002 April, May [2001-2002 points may be missed in our analysis due to confusion with S0-22, causing both their correlations to be below our detection threshhold]), S0-22 (1995 June, 1996 June, 1997 May, 1998 July, August, October, 1999 May, 2000 October, 2001 July, 2002 April, May [see note on S0-17]), S0-23 (1995 June, 2000 April, 2002 April, 2002 July - 2003 September), S0-25 (1995 June, 1996 June, 1998 April, July, 2000 July - 2001 May, 2002 April, 2002 July, 2003 April, September), and S0-26 (2000 April).

\footnotetext{
${ }^{\mathrm{f}}$ Measurements are dropped from the final proper motion fits as a result of significant astrometric biases due to nearby stars, as detailed in table footnote $d$ (except S0-2 in 1998 April and 1999 May) as well as measurements of S0-16 in 1998 August and S0-19 in 1999 July) or underestimated uncertainties (S0-4 in 1998 October and S0-5 in 2000 May). The procedure for identifying measurements to exclude is described in $\S 3.2$; both S0-3 and S0-21 have too few $(<3)$ points free from potential biases to carry out this procedure.
}

${ }^{g}$ These stars are independently identified in Schödel et al. (2003).

${ }^{\mathrm{h}} \mathrm{It}$ is difficult to assess explicitly why other measurements of S0-21 were not made, because of the lack of orbital information from only three measurement and the significant stellar confusion at its location. 
Table 2. Central Dark Mass Properties from Simultaneous Orbital Fit to Multiple Stars

\begin{tabular}{ll}
\hline \hline \multicolumn{1}{c}{ Parameter } & \multicolumn{1}{c}{ Estimated Value } \\
\hline Mass $\left(10^{6}\left(\frac{R_{o}}{8 k p c}\right)^{3} M_{\odot}\right)$ & $3.67 \pm 0.19$ \\
Position with Respect to S0-2 in 2003.0 (mas): & \\
$\Delta r_{R A}$ & $-36.5 \pm 1.6$ \\
$\Delta r_{D E C}$ & $-53.34 \pm 0.95$ \\
Proper Motion Relative to Central Cluster $\left(\right.$ mas y $\left.^{-1}\right):$ & \\
$V_{R A}$ & $0.87 \pm 0.46$ \\
$V_{D E C}$ & $1.16 \pm 0.57$ \\
\hline
\end{tabular}


Table 3. Stellar Orbital Parameters from Simultaneous Orbital Fit

\begin{tabular}{|c|c|c|c|c|c|c|c|c|c|}
\hline Star & $\begin{array}{c}P \\
(\mathrm{yrs})\end{array}$ & $\begin{array}{c}A^{\mathrm{a}} \\
(\mathrm{AU})\end{array}$ & $\begin{array}{c}T_{o} \\
(\mathrm{yrs})\end{array}$ & $e$ & $\begin{array}{c}i \\
(\mathrm{deg})\end{array}$ & $\begin{array}{r}\Omega^{\mathrm{b}} \\
(\mathrm{deg})\end{array}$ & $\begin{array}{c}\omega \\
(\operatorname{deg})\end{array}$ & $\begin{array}{c}q^{\mathrm{a}} \\
(\mathrm{AU})\end{array}$ & $\begin{array}{c}Q^{\mathrm{a}} \\
\left(\times 10^{3} \mathrm{AU}\right)\end{array}$ \\
\hline S0-2 & $14.53(0.65)$ & $919(23)$ & $2002.308(0.013)$ & $0.8670(0.0046)$ & $135.2(1.2)$ & $44.0(1.3)$ & $242.8(2.1)$ & $122.2(2.7)$ & $1.715(0.047)$ \\
\hline S0-16 & $36(17)$ & $1680(510)$ & $2000.201(0.025)$ & $0.974(0.016)$ & $102.7(2.2)$ & $44.8(1.9)$ & $155.9(3.8)$ & $45(16)$ & $3.3(1.0)$ \\
\hline S0-19 & $37.3(3.8)$ & $1720(110)$ & $1995.758(0.050)$ & $0.833(0.018)$ & $37.0(4.1)$ & $10.0(8.5)$ & $173.8(8.5)$ & $287(25)$ & $3.15(.22)$ \\
\hline S0-20 & $43(45)$ & $1900(1400)$ & $2005.4(3.6)$ & $0.40(0.21)$ & $23(38)$ & $66(49)$ & $260(100)$ & $1160(490)$ & $2.7(2.3)$ \\
\hline S0-1 & $190(180)$ & $5100(3200)$ & $1994.04(0.52)$ & $0.70(0.21)$ & $121.8(1.3)$ & $137.4(7.6)$ & $204(13)$ & $1530(180)$ & $8.7(6.5)$ \\
\hline S0-4 & $2600(130,000)$ & $30,000(950,000)$ & $1987.1(7.5)$ & $1.00(0.15)$ & $47(63)$ & $40(360)$ & $280(300)$ & $140(270)$ & $60(1,900)$ \\
\hline $\mathrm{S} 0-5$ & $9,900(430,000)$ & $70,000(2,100,000)$ & $2004.5(4.7)$ & $1.0(1.3)$ & $84.0(2.2)$ & $153.7(1.1)$ & $356(11)$ & $3206(79)$ & $100(4,200)$ \\
\hline
\end{tabular}

Note. - The first 3 stars represent a single joint solution. Each star below the line represents a four-star solution with the first 3 stars (see $\S 3.3 .1$ ). Values in parentheses are $1 \sigma$ uncertainties from the covariance matrix that takes into account the measurement uncertainties.

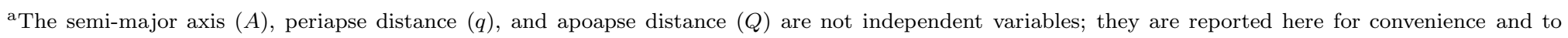
provide a proper accounting of their uncertainties. These quantities assume a distance of $8 \mathrm{kpc}$ and their uncertainties do not include the uncertainty associated with this distance.

${ }^{\mathrm{b}}$ The position angle of the nodal point is given for the node lying in the Eastern quadrants, except for S0-2 where it is the ascending node. 
Table 4. Absolute Astrometry of a Few Bright Sources Relative to Sgr A*- Radio

\begin{tabular}{lccccrc}
\hline \hline Source & $\begin{array}{c}t_{o} \\
(\text { year })\end{array}$ & $\begin{array}{c}\mathrm{R} \\
(\operatorname{arcsec})\end{array}$ & $\begin{array}{c}\Delta \mathrm{RA} \\
(\operatorname{arcsec})\end{array}$ & $\begin{array}{c}\Delta \mathrm{DEC} \\
(\operatorname{arcsec})\end{array}$ & $\begin{array}{c}\mathrm{v}_{R A} \\
\left(\mathrm{mas} \mathrm{y}^{-1}\right)\end{array}$ & \multicolumn{1}{c}{$\begin{array}{c}\mathrm{v}_{D E C} \\
\left(\mathrm{mas} \mathrm{y}^{-1}\right)\end{array}$} \\
\hline IRS 16NW & 2000.126 & 1.21 & $0.0183 \pm 0.0071$ & $1.212 \pm 0.014$ & $6.37 \pm 0.18$ & $1.47 \pm 0.12$ \\
IRS 16C & 2000.126 & 1.23 & $1.132 \pm 0.0069$ & $0.484 \pm 0.015$ & $-8.679 \pm 0.095$ & $7.32 \pm 0.12$ \\
\hline
\end{tabular}

